\title{
Genomic Context of the Two Integrons of ST-111 Pseudomonas aeruginosa AG1: A VIM-2-carrying Old-acquaintance and a Novel Imp-18-carrying Integron
}

Jose Arturo Molina-Mora ( $\nabla$ jose.molinamora@ucr.ac.cr)

Universidad de Costa Rica https://orcid.org/0000-0001-9764-4192

Diana Chinchilla-Montero

Universidad de Costa Rica

Raquel García Batán

Universidad de Costa Rica

Fernando García

Universidad de Costa Rica

\section{Research Article}

Keywords: Pseudomonas aeruginosa AG1, ST-111, IMP-18, VIM-2, pan-genome, genomic islands

Posted Date: December 31st, 2020

DOI: https://doi.org/10.21203/rs.3.rs-41474/v2

License: (c) (1) This work is licensed under a Creative Commons Attribution 4.0 International License.

Read Full License

Version of Record: A version of this preprint was published at Infection, Genetics and Evolution on April 1st, 2021. See the published version at https://doi.org/10.1016/j.meegid.2021.104740. 


\section{Abstract}

Pseudomonas aeruginosa is an opportunist and versatile organism responsible for infections mainly in immunocompromised hosts. This pathogen has high intrinsic resistance to most antimicrobials. $P$. aeruginosa AG1 (PaeAG1) is a Costa Rican high-risk ST-111 strain with resistance to multiple antibiotics, including carbapenems, due to the activity of VIM-2 and IMP-18 metallo- $\beta$-lactamases (MBLs). These genes are harbored in two class 1 integrons located inone out of the 57 PaeAG1 genomic islands. However, the genomic context associated to these determinants in PaeAG1 and other $P$. aeruginosa strains is unclear. Thus, we first assessed the transcriptional activity of VIM-2 and IMP-18 genes when exposed to imipenem (a carbapenem) by RT-qPCR. To select related genomes to PaeAG1, we implemented a pan-genome analysis to define and up-date the phylogenetic relationship among complete $P$. aeruginosa genomes. We also studied the PaeAG1 genomic islands content in the related strains and finally we described the architecture and possible evolutionary steps of the genomic regions around the VIM-2- and IMP-18-carrying integrons.

Expression of VIM-2 and IMP-18 genes was demonstrated to be induced after imipenem exposure. In a subsequent comparative genomics analysis with 211 strains, the $P$. aeruginosa pan-genome revealed that complete genome sequences are able to separate clones by MLST profile, including a clear ST-111 cluster with PaeAG1. The PaeAG1 genomic islands were found to define a diverse presence/absence pattern among related genomes. Finally, landscape reconstruction of genomic regions showed that VIM-2carrying integron (In59-like) is an old-acquaintance element harbored in the same known region found in other two ST-111 strains. Also, PaeAG1 has an exclusive genomic region containing a novel IMP-18carrying integron (registered as $\ln 1666$ ), with an arrangement never reported before. Altogether, we provide new insights about the genomic determinants associated with the resistance to carbapenems in this high-risk $P$. aeruginosa using comparative genomics.

\section{Introduction}

Pseudomonas aeruginosa is an opportunist and versatile pathogen able to survive in a wide variety of environments (Klockgether et al., 2010). With a large genome (6-7.5 Mb), a considerable proportion of the genome $(>8 \%)$ of $P$. aeruginosa strains is dedicated to regulatory functions (Cabot et al., 2016) resulting in a consequent diversity of metabolic capabilities and responses to stress (J. A. Molina-Mora et al., 2020; J. Molina-Mora et al., 2020). Due to these characteristics $P$. aeruginosa is responsible for infections among immunocompromised hosts (Lu et al., 2016) and nosocomial infections (Fernández, Corral-Lugo, \& Krell, 2018). This pathogen has high intrinsic resistance to most clinically used antimicrobials (Brazas, Brazas, Hancock, \& Hancock, 2005), many of them by multidrug-resistant or extensively drug-resistant strains (Oliver, Mulet, López-Causapé, \& Juan, 2015). This severely compromises the selection of appropriate treatments (X. Mulet et al., 2013) leading to a significant increase in the morbidity and mortality of these infections. According to World Health Organization (WHO), resistance to carbapenems in $P$. aeruginosa, Acinetobacter baumannii, and Enterobacteriaceae family is considered a critical issue in the context of antibiotic resistance, being classified as Priority 1 group (World Health Organization, 2017). 
$P$. aeruginosa AG1 (PaeAG1) is a particular $P$. aeruginosa strain isolated from an immunocompromised patient in a Costa Rican hospital in 2010 (Toval et al., 2015). This strain is resistant to multiple antibiotics such as $\beta$-lactams (including carbapenems), aminoglycosides, and fluoroquinolones, being only sensitive to colistin. This was the first reported strain of a $P$. aeruginosa isolate carrying both VIM-2 and IMP-18 genes encoding for metallo- $\beta$-lactamases (MBLs) enzymes, both with carbapenemase activity (Toval et al., 2015). As demonstrated in our previous works, including the genome assembly (GenBank CP045739) (J.-A. Molina-Mora, Campos-Sánchez, Rodríguez, Shi, \& García, 2020), these genes belong to two independent class 1 integrons, each contained in one out of the 57 predicted genomic islands of PaeAG1 (J.-A. Molina-Mora et al., 2020; Toval et al., 2015). Other elements such as six phages, mobile genetic elements, and some virulence factors are also harbored in genomic islands. Ciprofloxacin exposure in PaeAG1 induces phage activity with a very complex activity, affecting the growth in spite of this strain being resistant to this antibiotic (J. A. Molina-Mora et al., 2020). In addition, PaeAG1 has a not functional CRISPR-Cas system and molecular genotyping by multilocus sequence type (MLST) classifies PaeAG1 as a high-risk sequence type 111 (ST-111) strain (J.-A. Molina-Mora et al., 2020).

ST-111 is a lineage that belongs to the 012 serotype, including a multi-resistance profile and the ability to colonize nosocomial environments (X. Mulet et al., 2013; Turton et al., 2015; Witney et al., 2014; Woodford, Turton, \& Livermore, 2011). Toghether with ST-235 and ST-175 genotypes, ST-111 belongs to the high-risk group in $P$. aeruginosa (Oliver et al., 2015). High-risk clones are frequently associated with epidemics where multidrug resistance impairs thetreatment (Petitjean et al., 2017).

In this context, it is considered that $P$. aeruginosa high-risk clones are part of a non-clonal epidemic population structure (Oliver et al., 2015; Petitjean et al., 2017), many carrying genomic determinants such as carbapenemases or extended-spectrum $\beta$-lactamases (Oliver et al., 2015). Carbapenemases include Ambler class A enzymes such as KPC and GES variants, Ambler class B MBLs (IMP, VIM, SPM, GIM, NDM, and FIM type), and Amber class D (OXA variants) enzymes (Farajzadeh Sheikh et al., 2019; Hong et al., 2015). In Costa Rica, isolation of carbapenem-resistant $P$. aeruginosa strains is relatively common in some major hospitals as we reported, most of them carrying VIM or IMP alleles and up to a $63.1 \%$ prevalence (Toval et al., 2015). This is much higher than the frequencies observed in other countries (Hong et al., 2015).

VIM and IMP genes, as well as other MBLs, are frequently found as part of gene cassettes carried in integrons (Walsh, 2005; Zhao \& Hu, 2011), leading to the dissemination of multidrug resistance among Gram-negative bacteria (Jones-Dias et al., 2016). Thus, there is a growing interest in the reconstruction of the genomic context of mobile elements (in particular for integrons) to gain insights into bacterial evolution and its association with human activities, as well as to identify possible ways to mitigate antibiotic resistance (Ghaly, Chow, Asher, Waldron, \& Gillings, 2017). However, there are few studies about the genomic context of $P$. aeruginosa high-risk clones associated with integrons (Chowdhury et al., 2016).

In this sense, comparative genomic strategies can provide insights not only about gene content, architecture and evolutionary details, but also dynamics of mobile genetic elements, pathogenicity 
determinants, and others (Peter et al., 2019). Several studies at the genomic level have been implemented to describe the molecular diversity in $P$. aeruginosa (including high-risk clones) using different comparative approaches (Xavier Mulet et al., 2013; Petitjean et al., 2017; Turton et al., 2015). In our previous work, a pan-genome comparison was performed using only 11 ST-111 strains, in which the gene content was used to phylogenetically describe PaeAG1. However, as discussed there, an in-depth analysis including a significant number of strains (not only 11) is required to better understand the genomic features of PaeAG1 and other ST-111 strains (J.-A. Molina-Mora et al., 2020).

Since PaeAG1 has special genomic features regarding antibiotic multi-resistance, including VIM-2 and IMP-18 genes with carbapenemase activity, 57 genomic islands, and a ST-111 profile, we hypothesized that the comparative genomics can reveal insights about the evolution and landscape of genomic regions around the MBLs-carrying integrons of PaeAG1. Thus, the aim of the study was to compare the PaeAG1 genome against other $P$. aeruginosa sequences using comparative genomics to describe phylogenetic relationships, genomic islands content, and architecture of genomic regions associated with the VIM-2- and IMP-18-carrying integrons of PaeAG1. We first demonstrated that VIM-2 and IMP-18 are functional genes that can be induced after treatment with imipenem (a carbapenem antibiotic). We then analyzed all the complete $P$. aeruginosa genomes using a pan-genome analysis approach to identify related genomes to PaeAG1, revealing that whole genome sequences can separate clones by MLST profile (ST). Afterward, PaeAG1 genomic islands were searched in the related genomes, including all the ST-111 genomes, and diverse presence/absence patterns were found in related genomes. Finally, specific genomic regions associated with the two integrons were reconstructed and characterized to compare the gene content and architecture in close genomes. The genomic region associated with the VIM-2-carrying integron (In59-like) was completely found in other two ST-111 strains (i.e. it is an old-acquaintance integron), but an IMP-18-carrying integron (registered as $\ln 1666$ ), with an architecture never reported before, was found when the landscape of the related genomic region was described.

\section{Materials And Methods}

\subsection{Bacterial isolate}

The PaeAG1 strain is a Costa Rican isolate with resistance to $\beta$-lactams (including carbapenems, $\mathrm{MIC}_{\text {Imipenem }}>32 \mu \mathrm{g} / \mathrm{mL}$ ), aminoglycosides, and fluoroquinolones, being only sensitive to colistin. We recently assembled and annotated the PaeAG1 genome (J.-A. Molina-Mora et al., 2020) and data is available in Genbank under accession CP045739 (Bioproject PRJNA587210).

\subsection{RT-qPCR for VIM-2 and IMP-18 expression after imipenem exposure}

In order to study the expression of VIM-2 and IMP-18 genes by imipenem exposure in PaeAG1, experiments of growth curves and RT-qPCR were performed.

Growth curves assay: Three aliquots of pre-cultured PaeAG1 cells were added to fresh Lysogenic Broth (LB) broth to an initial optical density $\left(\mathrm{OD}_{600 \mathrm{~nm}}\right)$ of 0.01 . Each aliquot was treated with 0.0 (control), 25.0 
or $50.0 \mu \mathrm{g} / \mathrm{mL}$ of imipenem. Growth was monitored at times $0,2,4,6,8,12$, and 16 hours. The assay was performed in triplicates. Two specific aliquots at times 6 and 12 hours were taken for RT-qPCR assay, as follows.

RNA isolation: Aliquots at times 6 and 12 hours after imipenem exposure were preserved using the RNA protect reagent (QIAGEN). Total RNA was extracted using the RNeasy Mini kit (QIAGEN, UK) following the manufacturer's instructions. Subsequently, RNA was transcribed into cDNA with the Maxima H Minus First Strand cDNA Synthesis kit (Thermo Scientific ${ }^{\mathrm{TM}}$ Inc.). In the different steps, the quality and quantity of extracted RNA or cDNA were determined using a Nanodrop (Nanodrop 2000, Thermo Scientific ${ }^{\text {TM }}$ Inc.).

Primers sequences: Primers sequences for target VIM-2 and IMP-18 genes and the reference gene rpoD were previously described (Kim, Kim, \& Choi, 2003; Mendes et al., 2007; Savli et al., 2003). (Table 1). Primers were manufactured by Thermo Scientific ${ }^{\mathrm{TM}}$ Inc.

RT-qPCR: The standard curve method was implemented to quantify the expression of target and reference genes. Each reaction mixture contained $12.5 \mu \mathrm{L}$ of SYBR green Master Mix (Thermo Scientific ${ }^{\mathrm{TM}}$ Inc.), 0.25 $\mu \mathrm{L}$ of each primer, $10 \mu \mathrm{L}$ of PCR-grade water, and $2 \mu \mathrm{L}$ of cDNA. Thermocycling was performed in a StepOnePlus Real-Time PCR System (Thermo Scientific ${ }^{\text {TM }}$ Inc.). For VIM-2 and IMP-18 genes, the assay was run with denaturation at $95^{\circ} \mathrm{C}(10 \mathrm{~min}), 35 \mathrm{amplification}$ cycles of $94^{\circ} \mathrm{C}(20 \mathrm{~s}), 53^{\circ} \mathrm{C}(45 \mathrm{~s})$, and $72^{\circ} \mathrm{C}$ (30 s), with data acquisition at $72^{\circ} \mathrm{C}$. For the $r p o D$ gene, conditions were denaturation at $95^{\circ} \mathrm{C}(10 \mathrm{~min}), 45$ amplification cycles of $95^{\circ} \mathrm{C}(15 \mathrm{~s}), 20^{\circ} \mathrm{C}(10 \mathrm{~s})$, and $72^{\circ} \mathrm{C}(15 \mathrm{~s})$, with data acquisition at $72^{\circ} \mathrm{C}$. Melt curve data were used to determine the identity of the amplicons. .

Relative gene expression analysis: Gene expression of VIM-2 and IMP-18 in the experimental conditions $(0,25$, and $50 \mu \mathrm{g} / \mathrm{mL}$ imipenem) were normalized using the $r p o D$ housekeeping gene. Data were analyzed using the delta-delta Ct method (12). The change in gene expression within samples (time and antibiotic concentration) was calculated with reference to the control ( $0 \mu \mathrm{g} / \mathrm{mL}$ imipenem) and a two-way ANOVA test was performed between conditions (95\% confidence level).

\subsection{Datasets of complete $P$. aeruginosa genome sequences}

To compare all the complete genomic sequences of $P$. aeruginosa by a pan-genome analysis, metadata (including strain names, alternative ID, gene content, MLST profile, and others), genome and protein sequences (".fasta" format), and annotation (Genbank ".gbk" and ".tab" formats) files were retrieved from Pseudomonas Genomes Database (PGDB, https://pseudomonas.com).

\subsection{Comparative genomic analysis by a pan-genome approach}

Since differences in annotation were identified for many sequences, even in the same genomic regions, we decided to identify and annotate genes from the complete genomic sequences using the same approach. To achieve this, gene prediction and annotation were done using Prokka v1.13.3 (with -genus Pseudomonas -species aeruginosa and other parameters by default configuration) (Seemann, 2014). The Prokka annotation files (in ".gbk" format) were used to run the phylogenetic analysis by a pan- 
genome approach based on gene content in the Roary program v3.12.0 (Page et al., 2015) with default parameters. The phylogenetic tree (".newick" file) was visualized using Interactive Tree Of Life Tool (iTOL, https://itol.embl.de/) v5 (Letunic \& Bork, 2019), and strain names and MLST profiles were incorporated for each strain. For strains with unknown MLST, the profile was verified using the complete genome sequence approach (Larsen et al., 2012) in the MLST tool v2.0 (https://cge.cbs.dtu.dk/services/MLST/). For a functional analysis for all core-genes, STRINGdb (https://string-db.org/) was used to identify significantly enriched KEGG pathways (cutoff of false discovery rate FDR $<0.05$ ).

\subsection{Comparative analysis of the presence of PaeAG1 genomic islands in other strains}

The 57 PaeAG1 genomic islands were previously identified using IslandViewer v4 (www.pathogenomics.sfu.ca/islandviewer/), as we reported recently (J.-A. Molina-Mora et al., 2020). Regions of the genomic islands (".bed" file) were downloaded from the same platform and sequences (".fasta" format) were obtained using the getfasta function in bedtools software v2.29.2 (Quinlan \& Hall, 2010). The distribution of genomic islands along the genome was visualized using the BLAST Ring Image Generator BRIG tool v0.95 (Alikhan, Petty, Ben Zakour, \& Beatson, 2011).

In order to determine the presence and frequency of these genomic islands in other strains, a comparative analysis based on sequence alignment was done. Thus, we implemented a BLASTn pipeline to align PaeAG1 genomic island sequences and the complete genome sequences of all strains. A minimum length for coverage of $95 \%$ (overlap between query and subject sequences) and $80 \%$ of minimum sequence identity between sequences were used to define that a specific genomic island was present in a strain, similar to other studies (Holmes, Dallman, Shabaan, Hanson, \& Allison, 2018; Kluytmans-van den Bergh et al., 2016). A final comparison of the presence/absence of genomic islands was done for selected strains (see Results) using a small phylogenetic tree and a heatmap, which were visualized using phylo.heatmap function from phytools package v0.7-20 (https://www.rdocumentation.org/packages/phytools), in the R software (https://www.r-project.org/).

\subsection{Landscape of genomic regions associated with the two class 1 integrons of PaeAG1}

Two complete and independent class 1 integrons were previously identified in PaeAG1, one carrying the VIM-2 gene and another harboring the IMP-18 gene (J.-A. Molina-Mora et al., 2020). To better understand the possible evolutionary history of these integrons and their potential for lateral transfer, we reconstructed the genetic landscape of the genomic regions around these elements. The identity of the integrons was investigated using INTEGRALL database (http://integrall.bio.ua.pt). For the new integron (see Results), the same database was used for the registry and the integron number assignment.

Since the two integrons are absent in the reference strain Pae-PA01, an alignment of the genomic regions (BLASTn) and another of amino acid (AA) sequences (BLASTp) were used to identify the limits of the complete inserted region in PaeAG1. The two specific inserted regions were composed of two or more genomic islands in a row, as obtained in our previous study (grouped or with overlapping regions) (J.-A. 
Molina-Mora et al., 2020). Thus, regions were called GIC $\mathrm{VIM}_{-2}$ (genomic island cluster containing VIM-2carrying integron) and $\mathrm{GIC}_{\mathrm{IMP}-18}$ (genomic island cluster harboring the IMP-18-carrying integron).

A final alignment (BLASTn) of the extended regions (three coding genes on each side) of $\mathrm{GIC}_{\mathrm{VIM}-2}$ and $\mathrm{GIC}_{\text {IMP-18 }}$ was done against selected genomes. Genomes selection was done based on the phylogenetic relationships of strains close to PaeAG1 (pan-genome analysis) and the profile of presence/absence of the PaeAG1 genomic islands in other strains. All the syntenic regions of selected strains were compared using annotation files in Easyfig software v2.2.3 (Sullivan, Petty, \& Beatson, 2011), leading to visualize alignments, gene content and identity, exclusive/shared elements by strain and possible evolutionary steps, and others.

\section{Results}

\subsection{Expression of VIM-2 and IMP-18 genes is induced after imipenem treatment in PaeAG1}

In order to assess the functional activity of VIM-2 and IMP-18 genes, a RT-qPCR was performed. Exposure to imipenem had no effects on the growth curves of PaeAG1 (Fig. 1-A). Evaluation of gene expression after exposure to imipenem (Fig. 1-B-C) showed that VIM-2 and IMP-18 increased its expression at least by a 1.7 -fold (with respect to control) at 6 hours, but only 1.1 -fold at 12 hours. This observation was independent of the imipenem concentration ( 25 or $50 \mu \mathrm{g} / \mathrm{mL}$ ), as supported by the statistical analysis in which changes in the relative expression by time but not by concentration were significant for each gene.

3.2 Pan-genome analysis with the complete genome sequences defines P. aeruginosa clusters which correlates with the MLST genotyping profile

To select related genomes to PaeAG1, a total of 211 strains were analyzed to compare the genomic composition including PaeAG1 (Supplementary file 1 All_strains_information.xIsx contains the list of all the selected genomes, ID, strain, MLST profile, and others). Gene content comparison was done based on a pan-genome approach. A total of 2726 genes were identified as part of the core-genome (present $>99 \%$ strains). More details of results and complementary plots are provided in the Supplementary file 2 Pangenome analysis results.xlsx.

Enrichment analysis of KEGG pathways for all core genes (Table S1) found 42 biological processes implicated in several metabolism routes related to energy (carbon, fatty acids, amino acids), DNA and RNA, ribosomal activity, protein synthesis, and others.

As shown in Fig. 2, the similarity in the genomic composition by pan-genome analysis defines a phylogenetic tree able to separate groups that can be described in turn by the MLST genotyping profile. Although we identified a total of 67 different MLST profiles (and unknown cases), many of them resulted in low frequency. For example, 35 different ST classes had only a single strain (35 strains, $17 \%$ of all genomes) and 88 strains (42\%) belonged to the 56 ST profiles with less than five genomes. Also, 44 strains $(21 \%)$ had an allelic composition with an unknown ST profile. On the other hand, a total of 79 
(37\%) genomes corresponded to 11 ST classes with five or more strains. The last was evidenced using different colors by ST profile (as shown in Fig. 2), meanwhile strains belonging to low-frequency ST profiles were colored in the same way. Representative genomes such as the reference strain Pae-PAO1 (ST-549, purple cluster) and Pae-UCBPP-PA14 (ST-253, yellow group) were identified in the main ST groups.

PaeAG1 was located in the same group with the other nine ST-111 strains in a separated cluster (green). Other two ST profiles (low-frequency ST-234 and ST-654) and one unknown case (Pae-Pa84 strain) were kept close to this group. The whole group of these related strains, and the reference strain Pae-PA01, were used for subsequent analysis, including their phylogenetic relationships. For other high-risk clones, a single ST-175 genome was identified, and a clear cluster was found for the ten ST-235 genomes (including other genomes with unknown profiles).

3.3 Varying profiles of the presence/absence of the 57 PaeAG1 genomic islands are found in the ST-111 strains and related genomes

A comparative analysis based on sequence alignment was run to determine the presence and frequency of the PaeAG1 genomic islands in other phylogenetically related strains. Genomic islands locus were previously predicted (J.-A. Molina-Mora et al., 2020). We first represented the distribution of the genomic islands along the PaeAG1 genome, as presented in Fig. 3. Many of the islands are kept together, including overlapping regions or an arrangement in a row. Thus, we termed this as a genomic islands cluster (GIC) to refer to this group of islands. In Fig. 3, GICs correspond to the genomic regions labeled as joined names of the genomic islands, for example "GI48-49" represents the genomic region of islands GI48 and GI49. In some cases, each genomic island in the cluster can be differentially distributed in the genomes (for example GI48 is present in PaeAG1 and Pae-97, but GI49 is only found in PaeAG1, Fig. 4). For this reason, we do not re-define the locus neither joined the islands.

Analysis of the presence/absence of PaeAG1 genomic islands in other ST-111 strains and related genomes is shown in Fig. 4. Profiles for all the 211 is available in the Supplementary file 1 All_strains_information.xlsx, including total counts of strains by genomic islands, and total genomic islands per genome. The closest genomes to PaeAG1 (Pae-RIVM-EMC2982 and Pae-Carb0163) had the most similar profiles in the genomic islands content (41 genomic islands), but different patterns are obtained for other ST-111 strains. None of the islands is present in the reference genome Pae-PAO1, and other few genomic islands are rarely present in other non ST-111 strains.

On the other hand, two particular genomic islands were particularly recognized due to they carry the two PaeAG1 integrons. GI27 genomic island harbors the VIM-2-carrying integron, while IMP-18-carrying integron belongs to GI49. As shown in Fig. 4, GI27 (red) is present in PaeAG1 and two other ST-111 strains, and it is also absent in the rest of the 208 genomes. GI49 (blue) is unique to PaeAG1 and it is not it is present in none of the other 210 strains in the study. 
Additionally, both genomic islands are associated with a GIC, GI27-30 and GI48-49 (Fig. 3). Since the importance of these genomic regions to study the integrons, we specifically called them $\mathrm{GIC}_{\mathrm{VIM}-2}$ (genomic island cluster containing VIM-2-carrying integron) and $\mathrm{GIC}_{\mathrm{IMP}-18}$ (genomic island cluster harboring the IMP-18-carrying integron).

Based on phylogenetic relationships, ST profile, and genomic islands content, we selected specific genomes to compare the GICs associated with the integrons. As shown in Fig. 4, the four genomic islands of $\mathrm{GIC}_{\mathrm{VIM}-2}(\mathrm{Gl} 27-30)$ are differentially present in the genomes. For example, GI28 and G29 are present in eight strains, but GI27 in three and G30 in four. To specifically compare the genomic regions of $\mathrm{GIC}_{\mathrm{VIM}-2}$, we used the reference Pae-PA01, Pae-RIVM-EMC2982 (with the four genomic islands), and Pae-AR445 (with three of the genomic islands). For the case of $\mathrm{GIC}_{\mathrm{IMP}-18}$, the two islands $\mathrm{GI} 48$ and $\mathrm{GI} 49$ are absent in other ST-111 strains, but GI48 is present in Pae-97. Except for this case, no other strains in all 211 genomes were identified harboring both islands. To compare the genomic regions, the reference genome Pae-PA01, Pae-RIVM-EMC2982 as the closest genome, and Pae-97 (the only genome sharing a section of the GIC) were used.

\subsection{GIC $C_{V I M-2}$ is a known region containing the old-acquaintance VIM-2-carrying integron in PaeAG1}

With the aim of describing the possible evolutionary history of the VIM-2-carrying integron in PaeAG1, we described the architecture of the genomic regions delimited by the $\mathrm{GIC}_{\mathrm{VIM}-2}$ (including three genes on each side: 35798 bp and 32 protein-coding genes). Using Pae-PAO1 as reference, we found that genomic insertion occurred in the middle of the PA2229 gene, as shown in the top of Fig. 5. The insertion resulted mostly present in Pae-AR445 (coverage $94 \%$ and identity $99.97 \%$ of the PaeAG1 region), but without most of the integron (integrase int/1 and sul1 are present, unlike the gene cassette including VIM-2). However, a full coverage region was identified in Pae-RIV-EMC2982, with a $100 \%$ coverage and identity $99.99 \%$. The only two variants identified in the full region were non-synonymous mutations, with an amino-acid change in PaeAG1_03254 (transcriptional regulator merD, 99.0\% identity) and PaeAG1_03255 (mercuric reductase merA, 99.8\% identity). See Table 2 and Supplementary Table S2 for more details. Although not shown in Fig. 5, alignment was also done for Pae-Carb0163, which has the same profile of genomic islands content as Pae-RIV-EMC2982. In this case, a 100\% coverage and identity $99.87 \%$ (45 variants) were obtained in the $\mathrm{GIC}_{\mathrm{VIM}-2}$ region; most of the variants resulted in a change in the amino-acid sequence in PaeAG1_03245 (aacA29a, part of the integron with a 95.8\% identity resulting in aacA29e allele), but also affecting other three proteins (mercuric reductase, integrase Intl, and a transposase). See Supplementary Table S2 for more details.

Regarding the gene content (Table 2), this genomic insertion contains the complete integron carrying VIM2 gene. The composition of this integron is described in Fig. 5 (bottom), containing classical elements int1, attl, sul1, and the gene cassette (with aacA29a-b and VIM-2) of a class 1 integron, being classified as In59-like. Furthermore, $\mathrm{GIC}_{\mathrm{VIM}-2}$ has at least other mobile genetic elements, including transposases and recombinases modules. Other coding modules are associated with mercury metabolism or they remain 
unknown (hypothetical proteins). Details of the protein alignment of PaeAG1 against four genomes are also provided (Supplementary Table S2). Reconstruction of the evolutionary steps related to the conformation of this genomic region includes the participation of four transposons ( $\operatorname{Tn} 402, \operatorname{Tn} 21$-like, a disrupted and another complete Tn4661) as shown in Fig. 7-A. See details in Discussion.

Considering the full coverage and very high identity in at least two genomes, Pae-RIVM-EMC2982 and Pae-Carb0163, GIC $\mathrm{VIM}-2_{2}$ can be considered a genomic region present in two well-known VIM-2+ strains, being this gene located in an old-acquaintance class 1 integron (In59-like).

In the case of other VIM-2-carrying integrons, more than 90 elements have been reported (according to INTEGRALL database) and most of them include aacA alleles, as in the case of the In59-like integron present in PaeAG1 and other strains. A similar arrangement of this element is reported for other integrons such as $\ln 103, \operatorname{In} 927, \ln 1025$, and In1163. Details of all the $93 \mathrm{VIM}-2$-carrying integrons are described in Supplementary Table S3.

\subsection{GICIMP-18 is a PaeAG1 exclusive genomic region harboring a new IMP-18-carrying integron}

Similarly, we compared four genomes to describe the architecture of the genomic regions delimited by the $\mathrm{GIC}_{\mathrm{IMP}-18}$ (including three genes on each side: 30258 bp and 29 protein-coding genes). Using Pae-PAO1 as reference, we found that genomic insertion occurred between the genes PA4704 and PA4705, as shown in the top of Fig. 6. Genomic islands GI48-49 are absent in Pae-RIV-EMC2982 and Pae-Carb0163 genomes.

BLAST of $\mathrm{GIC}_{\mathrm{IMP}-18}$ identified the highest scored sequence in the Pae-97 genome (ST-234). Thus, since Pae-97 carries GI48, the syntenic comparison was done using this genome (Fig. 6). The analysis revealed a $77 \%$ coverage with identity $99.92 \%$. The Pae-97 integron also contains Int1, aacA genes, and another allele of the IMP gene (IMP-1), all with a different arrangement.

Regarding gene content (Table 3), this genomic insertion contains the complete integron carrying the IMP18 gene. The composition of this integron is described in Fig. 6 (bottom), containing int1, attl, sul1, and the gene cassette (IMP-18, gcuD, OXA-2, and aacA4). GIC $\mathrm{I}_{\mathrm{IMP}-18}$ also has genes coding for endonucleases and recombinases, or hypothetical proteins. Details of the protein alignment of PaeAG1 against the four genomes are also provided (see Supplementary Table S4).

Considering the absence of the complete region in other genomes and the first report of the architecture of this integron, $\mathrm{GIC}_{\mathrm{IMP}-18}$ can be considered a PaeAG1 exclusive region harboring a new IMP-18-carrying integron. This integron was registered as $\ln 1666$ in INTEGRALL database. According to the same database, at least seven IMP-18-carrying integrons have been reported. OXA, $g c u D$, aacA and/or aadA genes are frequently present with different arrangements, as shown in Table 4, including the new integron In1666. 
Conformation of $\mathrm{GIC}_{\mathrm{IMP}-18}$ region seems to include the participation of at least three mobile elements (the new integron In1666, insertion sequence IS1326, and transposon TnAs3) as shown in Fig. 7-B. However, a lack of information about the role of other elements (regions without matching sequences) makes it difficult to complete the possible evolutionary steps related to this genomic region.

In summary, the pan-genome analysis leads us to identify that the genomic content can separate groups according to the ST profile (MLST genotyping). All the ST-111 strains, including PaeAG1, resulted in the same phylogenetic group but different presence/absence profiles of PaeAG1 genomic islands were identified in other strains, even for grouped genomic islands, the GICs. Analysis of the landscape of regions $\mathrm{GIC}_{\mathrm{VIM}-2}$ and $\mathrm{GIC}_{\mathrm{IMP}-18}$ revealed one known and another new arrangement of genomic sequences in PaeAG1, harboring two independent MBLs-carrying integrons. The IMP-18-carrying integron has a unique and exclusive composition, reported here for the first time.

\section{Discussion}

Multi-resistance to antibiotics is a major threat to the public health because of the continuous emergence, worldwide spread, and increasing prevalence (Hong et al., 2015). With a high-risk ST-111 profile, PaeAG1 is a critical organism due to its resistance to multiple antibiotics, in particular to carbapenems (World Health Organization, 2017). In our previous work, the pan-genome analysis was focused on the gene content using only 11 genomes (ST-111) to phylogenetically compare PaeAG1 and other ST-111 strains (J.-A. Molina-Mora et al., 2020). In the current work, we included all the $P$. aeruginosa strains with complete genomes (211 strains) to update the core genome for this group, as well as we characterized the genomic islands and the genomic context of the two integrons, including the possible evolutionary steps that can explain the current state of the genomic architecture. Thus, these new results give more insights into the biology and evolution of the ST-111 strains, in particular for PaeAG1.

We first demonstrated that the expression of VIM-2 and IMP-18 genes (with carbapenemase activity) is induced after imipenem exposure, evidencing that are functional genes. A similar pattern in the expression levels, with an increment at 6 hours and a reduction at 12 hours, was found for both genes after treatment. Scarce studies have studied the expression patterns of various MBLs at the same time, including one recent study which found similar expression between VIM, IMP, and other MBL enzymes but depending on the sensitivity profile (Singh et al., 2019). Although the similar expression levels of VIM-2 and IMP-18 could be obtained by chance, a mechanistic relationship cannot be discarded. For example, an imipenem effect on PaeAG1 could be related to the induction of integrons as a response to stress. Cell stress after exposure to antibiotics is known to induce the SOS response, and the gene cassettes of the integrons can subsequently be induced (Hocquet et al., 2012). Since the two integrons of PaeAG1 are class 1 elements, they could be regulated similarly. More analyses are needed to validate or discard this possibility.

In the pan-genome analysis, we were able not only to reveal that 211 whole-genome sequences could separate clones by ST profile (MLST) but also the identification of core and accessory genes was 
achieved. Other pan-genome analyses in $P$. aeruginosa also found clusters that could be identified by the ST profile (Aguilar-Rodea et al., 2017; Weiser et al., 2019). While multiple comparative genomic analyses (many using a pan-genome approach) have been reported for $P$. aeruginosa (Aguilar-Rodea et al., 2017; Chowdhury et al., 2016; Freschi et al., 2019; Gomila, Peña, Mulet, Lalucat, \& García-Valdés, 2015; Hilker et al., 2015; Mosquera-Rendón et al., 2016; Ozer, Allen, \& Hauser, 2014; Poulsen et al., 2019; Valot et al., 2015; Weiser et al., 2019; Wendt \& Heo, 2016), most of them include incomplete, fragmented or draft genomes, or sequences of few genes. In 2015, complete genomes were used in a similar approach, but only 17 genomes were available (NCBI), and only three corresponded to high-risk clones (Valot et al., 2015). Thus, our analysis provides an update of the general status of relationships of the 211 available complete genomes by pan-genome analysis.

Regarding the gene content among all strains, we identified a total of 2726 genes as part of the coregenome (>99\% strains), similar to another similar approach (Mosquera-Rendón et al., 2016). Other studies have suggested a higher number of core genes (4000-5300) (Hilker et al., 2015; Ozer et al., 2014; Valot et al., 2015; Weiser et al., 2019) which are similar to our results with 4659 genes if we include the soft-coregenome (>95\% strains). Other cases using $>1000$ genomes have reported $<700$ core genes (Freschi et al., 2019; Poulsen et al., 2019). In general, differences can be explained by selected genomes (number, origin, completeness) and implemented algorithms (Hilker et al., 2015; Ozer et al., 2014).

In our approach, only complete genome sequences were used, contrasting with most of the other pangenome studies. Thus, the analyses were based on high confident sequences, which can be considered a strong point of our strategy. The previous study using complete genomes included the only 17 available sequences in 2015 (Valot et al., 2015). In the same line, another advantage of our approach is that we used the whole gene content, unlike other studies that have used few genes (Aguilar-Rodea et al., 2017) or variant analyses (Weiser et al., 2019) to relate strains. The last is a particular consideration for ubiquitous bacteria with a very plastic genome. A limitation of our approach is that the number of complete genomes is relatively small in comparison to the available draft genomes ( $>4500$ according to www.pseudomonas.com). Well-assembled draft genomes could be considered and more information could be generated, but a robust selection must be applied. However, that option was not considered here.

Biologically, the relatively high number of conserved genes in the core-genome can be associated with the ability to conquer multiple environments and to facilitate infectious capability towards a large set of hosts (Valot et al., 2015). According to functional analysis, 42 KEGG pathways (energy metabolism, nucleic acids, amino acids, ribosomal activity, and many others) were found as part of the enriched routes for all the core genes, with functions that are in line with other similar pan-genome studies (Mosquera-Rendón et al., 2016; Valot et al., 2015).

$P$. aeruginosa genome is composed of a mosaic structure including the large core-genome (Valot et al., 2015), into which regions of genomic plasticity lead to the insertion of a block of genes belonging to the accessory genome (Mathee et al., 2008). In the case of PaeAG1 and other ST-111 strains, the genome 
sequence is around 1.0 Mb longer that the reference genome Pae-PA01, a difference that is reflected as genomic islands distributed along the genome.

Pae-RIVM-EMC2982 and Pae-Carb0163 (closest genomes to PaeAG1) had the most similar profiles carrying 41 out of the genomic islands. As highlighted in the Results, many genomic islands formed clusters (GICs, Fig. 3), including the genomic islands clusters harboring the two integrons $\left(\mathrm{GIC}_{\mathrm{VIM}-2}\right.$ and $\left.\mathrm{GIC}_{\mathrm{IMP}-18}\right)$. Genomic island groups have been reported before as integrative and conjugative elements or ICEs (Petitjean et al., 2017), but ICEs in PaeAG1 (using ICEberg 2.0 platform, https://db$\mathrm{mml}$.sjtu.edu.cn/ICEfinder/ICEfinder.html) overlap with other $\mathrm{GICs}$ but none with $\mathrm{GIC}_{\mathrm{VIM}-2}$ and $\mathrm{GIC}_{\mathrm{IMP}-18}$. Since the size of the core-genome and its content is not well known (Valot et al., 2015), prediction methods are required to define accessory regions, but the outcome depends on algorithms (Ozer et al., 2014), which could explain differences and the GICs.

On the other hand, this prominent number of genomic islands in PaeAG1 and other ST-111 strains can be explained due to the absence of a functional CRISPR-Cas system (bacterial defense system against foreign DNA) and consequent high number of successful events of horizontal gene transfer (Petitjean et al., 2017). This genome plasticity of individual strains represents an advantage for $P$. aeruginosa to fit the needs for survival in virtually any environment (Mathee et al., 2008).

In the context of carbapenems resistance, genes encoding for MBLs are usually found as gene cassettes in class 1 integrons (Jones-Dias et al., 2016; Walsh, 2005). This allows rapid dissemination in the clinical setting due to the selective pressure by the use of antibiotics (Sánchez-Martinez et al., 2010), which is aggravated since this antibiotic represents the last therapeutic resource to treat $P$. aeruginosa infections (Toval et al., 2015). While multiple studies correlate antibiotic resistance and the presence of integrons, the genetic context surrounding class 1 integrons is often not investigated in $P$. aeruginosa, as remarked before (Chowdhury et al., 2016).

Carbapenem resistance in PaeAG1 was demonstrated to be explained by the activity of two MBLs (VIM-2 and IMP-18) (Toval et al., 2015), each gene harbored in two independent class 1 integrons (J.-A. MolinaMora et al., 2020; Toval et al., 2015).

Evaluation of the sequence showed that $\mathrm{GIC}_{\mathrm{VIM}-2}$ is also present in Pae-RIVM-EMC2982 $(100 \%$ coverage and $99.99 \%$ identity) and Pae-Carb0163 (100\% coverage and $99.87 \%$ identity) at the chromosomal level. However, a study including these strains showed that VIM-2-carrying integron and surrounding regions ( $\sim 30 \mathrm{~Kb}$, equivalent to $\mathrm{GIC}_{\mathrm{VIM}-2}$ ) were shared with a plasmid of ST-446 P. aeruginosa S04-90 with 99\% identity. Based on identity, mobilization of the fragment between plasmids and chromosomes may have occurred recently (van der Zee et al., 2018).

In the same study, analysis of genome landscape showed that the regions (equivalent to $\mathrm{GIC}_{\mathrm{VIM}-2}$ ) corresponded to a DNA segment acting as a composite transposon, composed of four different transposons (Tn402, Tn21-like, a disrupted and another complete Tn4661). The class 1 integron carrying 
VIM-2 is contained in the Tn402 transposon (Gillings, 2017; van der Zee et al., 2018). Evolutionary details are completely explained in (van der Zee et al., 2018). $\mathrm{GIC}_{\mathrm{VIM}-2}$ carries the genes involved in its transposition module (transposases such as TniB and TnpA) and mercury resistance module, as described in other similar transposons and insertion sequences (Chowdhury et al., 2016; Ghaly et al., 2017; Jones-Dias et al., 2016; Liebert, Hall, \& Summers, 1999; van der Zee et al., 2018). The presence of gene cassettes unrelated to the antibiotic resistance can be a result of anthropogenic settings (Ghaly et al., 2017) and selection pressures in environments polluted with heavy metals and other substances such as mercury, arsenic, and disinfectants (Gillings et al., 2015).

Regarding the VIM-2-carrying integron, this element is an In59-like integron. In59 was first reported two decades ago in France (Poirel et al., 2001) and then worldwide (Gillings, 2017; Samuelsen et al., 2010; Toval et al., 2015; van der Zee et al., 2018). Among all the 211 strains in our study, VIM-2 was only present in PaeAG1 and the two closest genomes (all ST-111). Differences in aacA29 genes defined the aacA29e allele found in Pae-Carb0163 (van der Zee et al., 2018), in contrast to aacA29a-b in PaeAG1, all coding for aminoglycoside acetyltransferases. In the case of other VIM-2-carrying integrons, most of the 93 reported elements have an aacA allele, including the case of the In59-like present in PaeAG1 and other strains. A similar arrangement has been reported in other elements including $\ln 103$, In927, In1025, and $\ln 1163$ (van der Zee et al., 2018).

Since $\mathrm{GIC}_{\mathrm{VIM}-2}$ sequence and architecture are completely found in two VIM-2+/ST-111 strains, VIM-2carrying integron (In59-like) can be considered an old-acquaintance element in a well-known genomic context.

Additionally, the genomic context defined by $\mathrm{GIC}_{\mathrm{IMP}-18}$ was also analyzed. Using Pae-PA01 as reference, it is shown that $\mathrm{GIC}_{\mathrm{IMP}-18}$ insertion occurred in a specific point (prrH) between PA4704 and PA4705 (Fig. 6). This region contains three genes for regulatory small RNAs ( $p r r F 1$, prrH, and prrF2) are found, which are involved in iron homeostasis under iron-depleted conditions (Reinhart et al., 2017) or to avoid iron toxicity (Reinhart et al., 2015).

While complete $\mathrm{GIC}_{\mathrm{IMP}-18}$ (composed of GI48-GI49 genomic islands) was found in none of the other strains, the GI48 section was found in the Pae-97 strain (ST-234, with a class 1 integron), a genome close to the ST-111 group (Fig. 2 and 3). Sequences comparison of $\mathrm{GIC}_{\mathrm{IMP}-18}$ and Pae- 97 showed $77 \%$ coverage and $99.92 \%$ identity. Gene composition of $\mathrm{GIC}_{\mathrm{IMP}-18}$ includes endonucleases and recombinases module, the class 1 integron, transposase TniB, and hypothetical proteins.

Concerning the integron harbored in $\mathrm{GIC}_{\mathrm{IMP}-18}$, the IMP-18-carrying element is composed of the int/1, the gene cassette (carrying IMP-18, gcuD, and OXA-2), aacA4, and sul1. Comparison of In1666 with other elements revealed that the new integron harbors other genes commonly found in other IMP-18-carrying elements, with a specific arrangement never reported before. The first report of IMP-18 in this bacterial group was the strain P. aeruginosa PS 297 (N Hanson, Hossain, Buck, Moland, \& Thomson, 2004; Nancy. Hanson, Hossain, Buck, Moland, \& Thomson, 2006). This North American isolate harbors an In98-like 
integron (called In133), including aacA7 and gcu39 alleles, contrasting the composition of other IMP-18carrying integrons (Borgianni et al., 2011), as shown in Table 4. Many of these integrons have been reported in Mexico (Garza-Ramos et al., 2008), Puerto Rico (Martínez, Vazquez, Aquino, Goering, \& Robledo, 2012), and United States (Nancy. Hanson et al., 2006), which are geographically related to this new report from Costa Rica.

In another strain, similar genes with another arrangement (orderly IMP-18, a disrupted aacA43, OXA-2, and gcuD) were reported for the first time in the In706 integron in 2012 (Martínez et al., 2012). Also, Pae-97 contains a class 1 integron, but with a different arrangement with IMP-1 allele (without OXA nor gcuD genes). Other studies found multiple strains carrying both IMP-18 and OXA-2 (without $g c u D$ nor aacA4) as part of In169 (Sánchez-Martinez et al., 2010) and In1215 (López-García et al., 2018) integrons, including some located in plasmids. Other integrons, such as $\ln 96, \ln 614$, and $\ln 707$, have other alleles and arrangements of the same genes (Martínez et al., 2012).

Since there is a lack of information about the genomic context of many IMP-carrying integrons (such as region $\mathrm{GIC}_{\mathrm{IMP}-18}$, unlike $\mathrm{GIC}_{\mathrm{VIM}-2}$ ), and the particular architecture of the class 1 integron in PaeAG1 with the gene cassette IMP-18/gcuD/OXA-2/aacA4, we consider that this IMP-18-carrying integron (registered as $\ln 1666)$ is a novel element that we report here for the first time.

In the partial reconstruction of the evolutionary steps related to the $\mathrm{GIC}_{\mathrm{IMP}-18}$ region, the integron $\operatorname{In} 1666$, the insertion sequence IS1326, and the transposon TnAs3 seem to play a key role in the current state of this genomic region. Both IS1326 and TnAs3 have been reported in different integrons and high plasticity regions (He et al., 2016; Jones-Dias et al., 2016; Liebert et al., 1999; Szuplewska, Czarnecki, \& Bartosik, 2014). Further analyses are required to complete the evolutionary steps which have defined this genomic region as well as the implications of multiresistant in PaeAG1.

Jointly, identification of the landscape of the genomic context defined by $\mathrm{GIC}_{\mathrm{VIM}-2}$ and $\mathrm{GIC}_{\mathrm{IMP}-18}$, provides insights about the dissemination and evolution of mobile elements, in this particular case for integrons carrying MBLs. Since MBL-producing $P$. aeruginosa is associated with epidemic outbreaks and contributes to the dissemination of carbapenemase resistance worldwide (Castanheira, Deshpande, Costello, Davies, \& Jones, 2014), it is worrisome that strains such as PaeAG1 circulate among Costa Rican hospitals. This can be correlated with the high prevalence of carbapenem-resistant strains in Costa Rica, many carrying VIM or IMP genes (Toval et al., 2015). Other ST-111 strains (harboring VIM-2 and IMP18) from major Costa Rican hospitals are being characterized, and the genomic context for these isolates (including the possible presence of the new integron In1666) will take part in further studies to describe the possible evolutionary steps and the mobilization of the high-risk strains between hospitals. Future works are necessary to contribute the surveillance system to evaluate if other circulating strains carry these two elements, to identify its possible dissemination, and lead an adequate infection control program in medical centers.

\section{Conclusions}


PaeAG1 is a high-risk and critical organism due to its resistance to carbapenems by the activity of VIM-2 and IMP-18 enzymes, both harbored in two class 1 integrons. To describe the genomic context associated with these integrons, we first verified the functionality of VIM-2 and IMP-18 after imipenem exposure. We then analyzed 211 complete genome sequences using a pan-genome analysis, separating strains by MLST profile. Analysis of the 57 PaeAG1 genomic islands showed a varying pattern of the presence/absence among all the strains, in particular for closest genomes to PaeAG1. Two selected genomic island clusters, $\mathrm{GIC}_{\mathrm{VIM}-2}$ and $\mathrm{GIC}_{\mathrm{IMP}-18}$, were studied in-depth. $\mathrm{GIC}_{\mathrm{VIM}-2}$ sequence was completely found in the other two known ST-111 strains, which contained the VIM-2-carrying integron as an oldacquaintance In59-like element. $\mathrm{GIC}_{\mathrm{IMP}-18}$ was partially found in another genome, but the IMP-18-carrying integron has an architecture never reported before, being considered as a novel In1666 integron. We provide new insights about the genomic determinants associated with this high-risk $P$. aeruginosa clone and its resistance to carbapenems using comparative genomics.

\section{Abbreviations}

FDR: False Discovery Rate

GI: Genomic island

GIC: Genomic islands cluster

IMP: Imipenemase

KEGG: Kyoto Encyclopedia of Genes and Genomes

MBLs: Metallo- $\beta$-lactamases

MLST: Multilocus sequence typing

PaeAG1: Pseudomonas aeruginosa AG1

ST: Sequence type

VIM: Verona integron-encoded MBLs

WHO: World Health Organization (WHO)

\section{Declarations}

Ethical approval and consent to participate

Not applicable. 
Not applicable.

\section{Availability of data and material}

All the strains we used in this study were obtained from NCBI. All the IDs are available in the "Supplementary_file 1 All_strains_information". For PaeAG1, the genome sequence and annotation files are available from our previous work at https://github.com/josemolina6/PaeAG1_genome, and in GenBank under the accession number CP045739.

\section{Declaration of Competing Interest}

The authors declare that there is no conflict of interest.

\section{Acknowledgments}

This work was funded by projects "B8114 Definición de la red transcriptómica y de las alteraciones genómicas inducidas por ciprofloxacina en Pseudomonas aeruginosa AG1", and "B8152 proNGS 1.0: Implementación y evaluación de protocolos de análisis de datos de tecnologías NGS y afines para el estudio de sistemas biológicos complejos", Vicerrectoría de Investigación, Universidad de Costa Rica (period 2017-2020).

We thank all members of Centro de Investigación en Enfermedades Tropicales (Universidad de Costa, Costa Rica) for their support in the activities associated with the projects.

\section{References}

Aguilar-Rodea, P., Zúñiga, G., Rodríguez-Espino, B. A., Cervantes, A. L. O., Arroyo, A. E. G., Moreno-Espinosa, S., ... Velázquez-Guadarrama, N. (2017). Identification of extensive drug resistant Pseudomonas aeruginosa strains: New clone ST1725 and high-risk clone ST233. PLOS ONE, 12(3), 2007-2013. https://doi.org/10.1371/journal.pone.0172882

Alikhan, N.-F., Petty, N. K., Ben Zakour, N. L., \& Beatson, S. A. (2011). BLAST Ring Image Generator (BRIG): simple prokaryote genome comparisons. BMC Genomics, 12(1), 402. https://doi.org/10.1186/1471-2164$12-402$

Borgianni, L., Prandi, S., Salden, L., Santella, G., Hanson, N. D., Rossolini, G. M., \& Docquier, J.-D. (2011). Genetic Context and Biochemical Characterization of the IMP-18 Metallo- $\beta$-Lactamase Identified in a Pseudomonas aeruginosa Isolate from the United States. Antimicrobial Agents and Chemotherapy, 55(1), 140-145. https://doi.org/10.1128/AAC.00858-10

Brazas, M. D., Brazas, M. D., Hancock, R. E. W., \& Hancock, R. E. W. (2005). Ciprofloxacin Induction of a Susceptibility Determinant in Pseudomonas aeruginosa. Antimicrobial Agents and Chemotherapy, 49(8), 3222-3227. https://doi.org/10.1128/AAC.49.8.3222 
Cabot, G., Zamorano, L., Moyà, B., Juan, C., Navas, A., Blázquez, J., \& Oliver, A. (2016). Evolution of Pseudomonas aeruginosa antimicrobial resistance and fitness under low and high mutation rates. Antimicrobial Agents and Chemotherapy, 60(3), 1767-1778. https://doi.org/10.1128/AAC.0267615.Address

Castanheira, M., Deshpande, L. M., Costello, A., Davies, T. A., \& Jones, R. N. (2014). Epidemiology and carbapenem resistance mechanisms of carbapenem-non-susceptible Pseudomonas aeruginosa collected during 2009-11 in 14 European and Mediterranean countries. Journal of Antimicrobial Chemotherapy, 69(7), 1804-1814. https://doi.org/10.1093/jac/dku048

Chowdhury, P. R., Scott, M., Worden, P., Huntington, P., Hudson, B., Karagiannis, T., ... Djordjevic, S. P. (2016). Genomic islands 1 and 2 play key roles in the evolution of extensively drug-resistant ST235 isolates of Pseudomonas aeruginosa. Open Biology, 6(3). https://doi.org/10.1098/rsob.150175

Farajzadeh Sheikh, A., Shahin, M., Shokoohizadeh, L., Halaji, M., Shahcheraghi, F., \& Ghanbari, F. (2019). Molecular epidemiology of colistin-resistant Pseudomonas aeruginosa producing NDM-1 from hospitalized patients in Iran. Iranian Journal of Basic Medical Sciences, 22(1), 38-42. https://doi.org/10.22038/ijbms.2018.29264.7096

Fernández, M., Corral-Lugo, A., \& Krell, T. (2018). The plant compound rosmarinic acid induces a broad quorum-sensing response in Pseudomonas aeruginosa PA01. Environmental Microbiology, 20(12), 4230-4244. https://doi.org/10.1111/1462-2920.14301

Freschi, L., Vincent, A. T., Jeukens, J., Emond-Rheault, J. G., Kukavica-Ibrulj, I., Dupont, M. J., ... Levesque, R. C. (2019). The Pseudomonas aeruginosa Pan-Genome Provides New Insights on Its Population Structure, Horizontal Gene Transfer, and Pathogenicity. Genome Biology and Evolution, 11(1), 109-120. https://doi.org/10.1093/gbe/evy259

Garza-Ramos, U., Tinoco, P., Silva-Sanchez, J., Morfin-Otero, R., Rodriguez-Noriega, E., Leon-Garnica, G., ... Jones, R. N. (2008). Metallo- $\beta$-lactamase IMP-18 is located in a class 1 integron (In96) in a clinical isolate of Pseudomonas aeruginosa from Mexico. International Journal of Antimicrobial Agents, 31(1), 78-80. https://doi.org/10.1016/j.ijantimicag.2007.08.003

Ghaly, T. M., Chow, L., Asher, A. J., Waldron, L. S., \& Gillings, M. R. (2017). Evolution of class 1 integrons: Mobilization and dispersal via food-borne bacteria. PLOS ONE, 12(6), 1-11.

https://doi.org/10.1371/journal.pone.0179169

Gillings, M. R. (2017). Class 1 integrons as invasive species. Current Opinion in Microbiology, 38, 10-15. https://doi.org/10.1016/j.mib.2017.03.002

Gillings, M. R., Gaze, W. H., Pruden, A., Smalla, K., Tiedje, J. M., \& Zhu, Y.-G. (2015). Using the class 1 integron-integrase gene as a proxy for anthropogenic pollution. The ISME Journal, 9(6), 1269-1279. https://doi.org/10.1038/ismej.2014.226 
Gomila, M., Peña, A., Mulet, M., Lalucat, J., \& García-Valdés, E. (2015). Phylogenomics and systematics in Pseudomonas. Frontiers in Microbiology, 6(MAR), 1-13. https://doi.org/10.3389/fmicb.2015.00214

Hanson, N, Hossain, A., Buck, L., Moland, E., \& Thomson, K. (2004). IMP-18. Presented at the 44th Interscience Conference on Antimicrobial Agents and Chemotherapy, 64. Washington, USA.

Hanson, Nancy., Hossain, A., Buck, L., Moland, E. S., \& Thomson, K. S. (2006). First Occurrence of a Pseudomonas aeruginosa Isolate in the United States Producing an IMP Metallo- $\beta$-Lactamase, IMP-18. Antimicrobial Agents and Chemotherapy, 50(6), 2272. https://doi.org/10.1128/AAC.01440-05

He, S., Chandler, M., Varani, A. M., Hickman, A. B., Dekker, J. P., \& Dyda, F. (2016). Mechanisms of evolution in high-consequence drug resistance plasmids. MBio, 7(6), 1987-2003.

https://doi.org/10.1128/mBio.01987-16

Hilker, R., Munder, A., Klockgether, J., Losada, P. M., Chouvarine, P., Cramer, N., ... Tümmler, B. (2015). Interclonal gradient of virulence in the $P$ seudomonas aeruginosa pangenome from disease and environment. Environmental Microbiology, 17(1), 29-46. https://doi.org/10.1111/1462-2920.12606

Hocquet, D., Llanes, C., Thouverez, M., Kulasekara, H. D., Bertrand, X., Plésiat, P., ... Miller, S. I. (2012). Evidence for induction of integron-based antibiotic resistance by the SOS response in a clinical setting. PLoS Pathogens, 8(6). https://doi.org/10.1371/journal.ppat.1002778

Holmes, A., Dallman, T. J., Shabaan, S., Hanson, M., \& Allison, L. (2018). Validation of whole-genome sequencing for identification and characterization of Shiga toxin-producing Escherichia coli to produce standardized data to enable data sharing. Journal of Clinical Microbiology, 56(3). https://doi.org/10.1128/JCM.01388-17

Hong, D. J., Bae, I. K., Jang, I. H., Jeong, S. H., Kang, H. K., \& Lee, K. (2015). Epidemiology and characteristics of metallo-ß-lactamase-producing Pseudomonas aeruginosa. Infection and Chemotherapy, 47(2), 81-97. https://doi.org/10.3947/ic.2015.47.2.81

Jones-Dias, D., Manageiro, V., Ferreira, E., Barreiro, P., Vieira, L., Moura, I. B., \& Caniça, M. (2016). Architecture of class 1,2, and 3 integrons from gram negative bacteria recovered among fruits and vegetables. Frontiers in Microbiology, 7(SEP), 1-13. https://doi.org/10.3389/fmicb.2016.01400

Kim, S.-M., Kim, E.-C., \& Choi, S.-Y. (2003). Typing by Pulsed Field Gel Electrophoresis and Detection of Metallo- $\beta$-lactamase Gene Against Acinetobacter baumannii from Clinical Specimens. Korean J Clin Lab Sci, 35(2), 90-98. Retrieved from http://www.kjcls.org/journal/view.html?

spage $=90 \&$ volume $=35 \&$ number $=2$

Klockgether, J., Munder, A., Neugebauer, J., Davenport, C. F., Stanke, F., Larbig, K. D., ... Tümmler, B. (2010). Genome diversity of Pseudomonas aeruginosa PA01 laboratory strains. Journal of Bacteriology, 192(4), 1113-1121. https://doi.org/10.1128/JB.01515-09 
Kluytmans-van den Bergh, M. F., Huizinga, P., Bonten, M. J., Bos, M., De Bruyne, K., Friedrich, A. W., ... Kluytmans, J. A. (2016). Presence of mcr-1 -positive Enterobacteriaceae in retail chicken meat but not in humans in the Netherlands since 2009. Eurosurveillance, 21(9), 30149. https://doi.org/10.2807/15607917.ES.2016.21.9.30149

Larsen, M. V, Cosentino, S., Rasmussen, S., Friis, C., Hasman, H., Marvig, R. L., ... Lund, O. (2012). Multilocus sequence typing of total-genome-sequenced bacteria. Journal of Clinical Microbiology, 50(4), 1355-1361. https://doi.org/10.1128/JCM.06094-11

Letunic, I., \& Bork, P. (2019). Interactive Tree Of Life (iTOL) v4: recent updates and new developments. Nucleic Acids Research, 47(W1), W256-W259. https://doi.org/10.1093/nar/gkz239

Liebert, C. A., Hall, R. M., \& Summers, A. O. (1999). Transposon Tn21, Flagship of the Floating Genome. Microbiology and Molecular Biology Reviews, 63(3), 507-522. https://doi.org/10.1128/mmbr.63.3.507522.1999

López-García, A., Rocha-Gracia, R. del C., Bello-López, E., Juárez-Zelocualtecalt, C., Sáenz, Y., CastañedaLucio, M., ... Lozano-Zarain, P. (2018). Characterization of antimicrobial resistance mechanisms in carbapenem-resistant Pseudomonas aeruginosa carrying IMP variants recovered from a Mexican Hospital. Infection and Drug Resistance, 11, 1523. https://doi.org/10.2147/IDR.S173455

Lu, P., Wang, Y., Zhang, Y., Hu, Y., Thompson, K. M., \& Chen, S. (2016). RpoS-dependent sRNA RgsA regulates Fis and AcpP in Pseudomonas aeruginosa. Molecular Microbiology, 102(2), 244-259. https://doi.org/10.1111/mmi.13458

Martínez, T., Vazquez, G. J., Aquino, E. E., Goering, R. V, \& Robledo, I. E. (2012). Two novel class I integron arrays containing IMP-18 metallo- $\beta$-lactamase gene in Pseudomonas aeruginosa clinical isolates from Puerto Rico. Antimicrobial Agents and Chemotherapy, 56(4), 2119-2121.

https://doi.org/10.1128/AAC.05758-11

Mathee, K., Narasimhan, G., Valdes, C., Qiu, X., Matewish, J. M., Koehrsen, M., ... Lory, S. (2008). Dynamics of Pseudomonas aeruginosa genome evolution. Proceedings of the National Academy of Sciences, 105(8), 3100-3105. https://doi.org/10.1073/PNAS.0711982105

Mendes, R. E., Kiyota, K. A., Monteiro, J., Castanheira, M., Andrade, S. S., Gales, A. C., ... Tufik, S. (2007). Rapid detection and identification of metallo- $\beta$-lactamase-encoding genes by multiplex real-time PCR assay and melt curve analysis. Journal of Clinical Microbiology, 45(2), 544-547.

https://doi.org/10.1128/JCM.01728-06

Molina-Mora, J.-A., Campos-Sánchez, R., Rodríguez, C., Shi, L., \& García, F. (2020). High quality 3C de novo assembly and annotation of a multidrug resistant ST-111 Pseudomonas aeruginosa genome: Benchmark of hybrid and non-hybrid assemblers. Scientific Reports, 10(1), 1392. https://doi.org/10.1038/s41598020-58319-6 
Molina-Mora, J. A., Chinchilla, D., Chavarría, M., Ulloa, A., Campos-Sanchez, R., Mora-Rodríguez, R. A., ... García, F. (2020). Transcriptomic determinants of the response of ST-111 Pseudomonas aeruginosa AG1 to ciprofloxacin identified by a top-down systems biology approach. Scientific Reports, 10, 1-23. https://doi.org/10.1038/s41598-020-70581-2

Molina-Mora, J., Montero-Manso, P., Batán, R. G., Sánchez, R. C., Fernández, J. V., \& García, F. (2020). A first Pseudomonas aeruginosa perturbome: Identification of core genes related to multiple perturbations by a machine learning approach. BioRxiv, 2020.05.05.078477. https://doi.org/10.1101/2020.05.05.078477

Mosquera-Rendón, J., Rada-Bravo, A. M., Cárdenas-Brito, S., Corredor, M., Restrepo-Pineda, E., \& BenítezPáez, A. (2016). Pangenome-wide and molecular evolution analyses of the Pseudomonas aeruginosa species. BMC Genomics, 17(1), 1-14. https://doi.org/10.1186/s12864-016-2364-4

Mulet, X., Cabot, G., Ocampo-Sosa, A. A., Dominguez, M. A., Zamorano, L., Juan, C., ... Spanish Network for Research in Infectious Diseases (REIPI). (2013). Biological Markers of Pseudomonas aeruginosa Epidemic High-Risk Clones. Antimicrobial Agents and Chemotherapy, 57(11), 5527-5535. https://doi.org/10.1128/AAC.01481-13

Mulet, Xavier, Cabot, G., Ocampo-Sosa, A. A., Dominguez, M. A., Zamorano, L., Juan, C., ... Oliver, A. (2013). Biological markers of Pseudomonas aeruginosa epidemic high-risk clones. Antimicrobial Agents and Chemotherapy, 57(11), 5527-5535. https://doi.org/10.1128/AAC.01481-13

Oliver, A., Mulet, X., López-Causapé, C., \& Juan, C. (2015). The increasing threat of Pseudomonas aeruginosa high-risk clones. Drug Resistance Updates, 21-22, 41-59.

https://doi.org/10.1016/j.drup.2015.08.002

Ozer, E. A., Allen, J. P., \& Hauser, A. R. (2014). Characterization of the core and accessory genomes of Pseudomonas aeruginosa using bioinformatic tools Spine and AGEnt. BMC Genomics, 15(1), 737. https://doi.org/10.1186/1471-2164-15-737

Page, A. J., Cummins, C. A., Hunt, M., Wong, V. K., Reuter, S., Holden, M. T. G., ... Parkhill, J. (2015). Roary: rapid large-scale prokaryote pan genome analysis. Bioinformatics, 31(22), 3691-3693. https://doi.org/10.1093/bioinformatics/btv421

Peter, S., Bosio, M., Gross, C., Bezdan, D., Gutierrez, J., Oberhettinger, P., ... Ossowski, S. (2019). Tracking of antibiotic resistance transfer and rapid plasmid evolution in a hospital setting by Nanopore sequencing. BioRxiv, 639609. https://doi.org/10.1101/639609

Petitjean, M., Martak, D., Silvant, A., Bertrand, X., Valot, B., \& Hocquet, D. (2017). Genomic characterization of a local epidemic Pseudomonas aeruginosa reveals specific features of the widespread clone ST395. Microbial Genomics, 3(10), e000129. https://doi.org/10.1099/mgen.0.000129 
Poirel, L., Lambert, T., Turkoglu, S., Ronco, E., Gaillard, J., \& Nordmann, P. (2001). Characterization of Class 1 Integrons from Pseudomonas aeruginosa That Contain the blaVIM-2 Carbapenem-Hydrolyzing Lactamase Gene and of Two Novel Aminoglycoside Resistance Gene Cassettes. Antimicrobial Agents and Chemotherapy, 45(2), 546-552. https://doi.org/10.1128/AAC.45.2.546-552.2001

Poulsen, B. E., Yang, R., Clatworthy, A. E., White, T., Osmulski, S. J., Li, L., ... Hung, D. T. (2019). Defining the core essential genome of Pseudomonas aeruginosa. Proceedings of the National Academy of Sciences of the United States of America, 116(20), 10072-10080. https://doi.org/10.1073/pnas.1900570116

Quinlan, A. R., \& Hall, I. M. (2010). BEDTools: a flexible suite of utilities for comparing genomic features. Bioinformatics, 26(6), 841-842. https://doi.org/10.1093/bioinformatics/btq033

Reinhart, A. A., Nguyen, A. T., Brewer, L. K., Bevere, J., Jones, J. W., Kane, M. A., ... Oglesby-Sherrouse, A. G. (2017). The Pseudomonas aeruginosa PrrF Small Acute Murine Lung Infection. Infection and Immunity, 85(5), 1-15. https://doi.org/10.1128/IAl.00764-16

Reinhart, A. A., Powell, D. A., Nguyen, A. T., O’Neill, M., Djapgne, L., Wilks, A., ... Oglesby-Sherrouse, A. G. (2015). The prrF-encoded small regulatory RNAs are required for iron homeostasis and virulence of Pseudomonas aeruginosa. Infection and Immunity, 83(3), 863-875. https://doi.org/10.1128/IAl.0270714

Samuelsen, Ø., Toleman, M. A., Sundsfjord, A., Rydberg, J., Leegaard, T. M., Walder, M., ... Giske, C. G. (2010). Molecular epidemiology of metallo- $\beta$-lactamase-producing Pseudomonas aeruginosa isolates from Norway and Sweden shows import of international clones and local clonal expansion. Antimicrobial Agents and Chemotherapy, 54(1), 346-352. https://doi.org/10.1128/AAC.00824-09

Sánchez-Martinez, G., Garza-Ramos, U. J., Reyna-Flores, F. L., Gaytán-Martínez, J., Lorenzo-Bautista, I. G., \& Silva-Sanchez, J. (2010). In169, A New Class 1 Integron that Encoded blaIMP-18 in a MultidrugResistant Pseudomonas aeruginosa Isolate from Mexico. Archives of Medical Research, 41(4), 235-239. https://doi.org/10.1016/j.arcmed.2010.05.006

Savli, H., Karadenizli, A., Kolayli, F., Gundes, S., Ozbek, U., \& Vahaboglu, H. (2003). Expression stability of six housekeeping genes: a proposal for resistance gene quantification studies of Pseudomonas aeruginosa by real-time quantitative RT-PCR. Journal of Medical Microbiology, 52(5), 403-408. https://doi.org/10.1099/jmm.0.05132-0

Seemann, T. (2014). Prokka: rapid prokaryotic genome annotation. Bioinformatics, 30(14), 2068-2069. https://doi.org/10.1093/bioinformatics/btu153

Singh, T., Singh, P. K., Das, S., Wani, S., Jawed, A., \& Dar, S. A. (2019). Transcriptome analysis of betalactamase genes in diarrheagenic Escherichia coli. Scientific Reports, 9(1).

https://doi.org/10.1038/s41598-019-40279-1 
Sullivan, M. J., Petty, N. K., \& Beatson, S. A. (2011). Easyfig: a genome comparison visualizer. Bioinformatics, 27(7), 1009. https://doi.org/10.1093/BIOINFORMATICS/BTR039

Szuplewska, M., Czarnecki, J., \& Bartosik, D. (2014). Autonomous and non-autonomous Tn 3 -family transposons and their role in the evolution of mobile genetic elements . Mobile Genetic Elements, 4(6), 14. https://doi.org/10.1080/2159256x.2014.998537

Toval, F., Guzmán-Marte, A., Madriz, V., Somogyi, T., Rodríguez, C., \& García, F. (2015). Predominance of carbapenem-resistant Pseudomonas aeruginosa isolates carrying blaIMP and blaVIM metallo- $\beta$ lactamases in a major hospital in Costa Rica. Journal of Medical Microbiology, 64(1), 37-43. https://doi.org/10.1099/jmm.0.081802-0

Turton, J. F., Wright, L., Underwood, A., Witney, A. A., Chan, Y. T., Al-Shahib, A., ... Woodford, N. (2015). Highresolution analysis by whole-genome sequencing of an international lineage (Sequence Type 111) of pseudomonas aeruginosa associated with metallo-carbapenemases in the United Kingdom. Journal of Clinical Microbiology, 53(8), 2622-2631. https://doi.org/10.1128/JCM.00505-15

Valot, B., Guyeux, C., Rolland, J. Y., Mazouzi, K., Bertrand, X., \& Hocquet, D. (2015). What It Takes to Be a Pseudomonas aeruginosa? The Core Genome of the Opportunistic Pathogen Updated. PLOS ONE, 1O(5), e0126468. https://doi.org/10.1371/journal.pone.0126468

van der Zee, A., Kraak, W. B., Burggraaf, A., Goessens, W. H. F., Pirovano, W., Ossewaarde, J. M., \& Tommassen, J. (2018). Spread of carbapenem resistance by transposition and conjugation among Pseudomonas aeruginosa. Frontiers in Microbiology, 9(SEP), 1-11.

https://doi.org/10.3389/fmicb.2018.02057

Walsh, T. R. (2005). The emergence and implications of metallo- $\beta$-lactamases in Gram-negative bacteria. Clinical Microbiology and Infection, Supplement, 11(6), 2-9. https://doi.org/10.1111/j.14690691.2005.01264.x

Weiser, R., Green, A. E., Bull, M. J., Cunningham-Oakes, E., Jolley, K. A., Maiden, M. C. J., ... Mahenthiralingam, E. (2019). Not all pseudomonas aeruginosa are equal: Strains from industrial sources possess uniquely large multireplicon genomes. Microbial Genomics, 5(7).

https://doi.org/10.1099/mgen.0.000276

Wendt, M., \& Heo, G.-J. (2016). Multilocus sequence typing analysis of Pseudomonas aeruginosa isolated from pet Chinese stripe-necked turtles ( Ocadia sinensis ) . Laboratory Animal Research, 32(4), 208. https://doi.org/10.5625/lar.2016.32.4.208

Witney, A. A., Gould, K. A., Pope, C. F., Bolt, F., Stoker, N. G., Cubbon, M. D., ... Hinds, J. (2014). Genome sequencing and characterization of an extensively drug-resistant sequence type 111 serotype 012 hospital outbreak strain of Pseudomonas aeruginosa. Clinical Microbiology and Infection, 20(10), 06090618. https://doi.org/10.1111/1469-0691.12528

Page 23/36 
Woodford, N., Turton, J. F., \& Livermore, D. M. (2011). Multiresistant Gram-negative bacteria: the role of high-risk clones in the dissemination of antibiotic resistance. FEMS Microbiology Reviews, 35(5), 736755. https://doi.org/10.1111/j.1574-6976.2011.00268.x

World Health Organization. (2017). Guidelines for the prevention and control of carbapenem-resistant Enterobacteriaceae, Acinetobacter baumannii and Pseudomonas aeruginosa in health care facilities. Retrieved from https://apps.who.int/iris/bitstream/handle/10665/259462/9789241550178-eng.pdf? sequence $=1 \&$ ua $=1$

Zhao, W. H., \& Hu, Z. Q. (2011). IMP-type metallo- $\beta$-lactamases in Gram-negative bacilli: Distribution, phylogeny, and association with integrons. Critical Reviews in Microbiology, 37(3), 214-226. https://doi.org/10.3109/1040841X.2011.559944

\section{Tables}

Table 1. Primer sequences used for RT-qPCR experiments.

\begin{tabular}{c|c|c|c|c}
\hline Gene & Primer & Sequence $\left(5^{\prime} \rightarrow 3^{\prime}\right)$ & Final concentration & Amplicon length \\
\hline \multirow{2}{*}{ IMP-18 } & Forward & GAATAG(A/G) $(\mathrm{A} / \mathrm{G})$ TGGCTTAA(C/T)TCTC & $1 \mu \mathrm{M}$ & $188 \mathrm{pb}$ \\
\cline { 2 - 3 } & Reverse & CCAAAC $(\mathrm{C} / \mathrm{T})$ ACTA(G/C)GTTATC & & \\
\hline \multirow{2}{*}{ VIM-2 } & Forward & CCGCGTCTATCATGGCTATT & $0.1 \mu \mathrm{M}$ & $181 \mathrm{pb}$ \\
\cline { 2 - 3 } & Reverse & ATGAGACCATTGGACGGGTA & & \\
\hline \multirow{2}{*}{$r p o D$} & Forward & GGGCGAAGAAGGAAATGGTC & $1 \mu \mathrm{M}$ & $178 \mathrm{pb}$ \\
\cline { 2 - 3 } & Reverse & CAGGTGGCGTAGGTGGAGAA & & \\
\hline
\end{tabular}

Table 2. Annotation of protein-coding genes of the genomic region $\mathrm{GIC}_{\mathrm{VIM}-2}$ associated with the VIM-2-carrying integron 


\begin{tabular}{|c|c|c|c|c|c|}
\hline \multirow[t]{2}{*}{ PaeAG1 } & \multicolumn{2}{|c|}{ Pae-RIV-EMC2982 } & \multirow[t]{2}{*}{ Annotation } & \multirow[t]{2}{*}{ Name } & \multirow[t]{2}{*}{ RefSeq } \\
\hline & Gene* & Identity & & & \\
\hline PaeAG1_03237 & EMC2982_03491 & 100.0 & $\begin{array}{l}\text { PslA, psl cluster plays a role in cell-cell } \\
\text { and/or cell-surface interaction in biofilm } \\
\text { formation }\end{array}$ & pslA (PA2231) & $\begin{array}{c}\text { NP_250921.1; } \\
\text { WP_003111160.1 }\end{array}$ \\
\hline PaeAG1_03238 & EMC2982_03490 & 100.0 & Hypothetical protein PA2230 & PA2230 & $\begin{array}{c}\text { NP_250920.1; } \\
\text { WP_003122761.1 }\end{array}$ \\
\hline PaeAG1_03239 & EMC2982_03489 & 100.0 & Hypothetical protein PA2229 & PA2229 & $\begin{array}{c}\text { NP_250919.1; } \\
\text { WP_003113716.1 }\end{array}$ \\
\hline PaeAG1_03240 & EMC2982_03488 & 100.0 & Hypothetical protein & $\mathrm{HP}$ & WP_034066849.1 \\
\hline PaeAG1_03241 & EMC2982_03487 & 100.0 & Transposase TnpA & $\operatorname{tnpA}$ & WP_003460108.1 \\
\hline PaeAG1_03242 & EMC2982_03486 & 100.0 & Transposase TnpR & $\operatorname{tnpR}$ & $\begin{array}{l}\text { WP_000147567.1; } \\
\text { YP_005211182.1 }\end{array}$ \\
\hline PaeAG1_03243 & EMC2982_03485 & 100.0 & Transposase TnpM & $\operatorname{tnpM}$ & WP_004217866.1 \\
\hline PaeAG1_03244 & EMC2982_03484 & 100.0 & Class I integron integrase IntI & intI & YP_005221021.1 \\
\hline PaeAG1_03245 & EMC2982_03483 & 100.0 & $\begin{array}{c}\text { 6'-N-aminoglycoside acetyltransferase } \\
\text { type I aacA29a }\end{array}$ & aacA29a & WP_032490447.1 \\
\hline PaeAG1_03246 & EMC2982_03482 & 100.0 & $\begin{array}{c}\text { Carbapenem-hydrolyzing metallo-beta- } \\
\text { lactamase VIM-2 }\end{array}$ & VIM-2 & WP_032491390.1 \\
\hline PaeAG1_03247 & EMC2982_03481 & 100.0 & $\begin{array}{c}\text { 6'-N-aminoglycoside acetyltransferase } \\
\text { type I aacA29b }\end{array}$ & aacA29b & WP_032490447.1 \\
\hline PaeAG1_03248 & EMC2982_03480 & 100.0 & $\begin{array}{l}\text { Sulfonamide-resistant dihydropteroate } \\
\text { synthase Sul1 }\end{array}$ & sul1 & WP_000259031.1 \\
\hline PaeAG1_03249 & EMC2982_03479 & 100.0 & Acetyltransferase & Acetyltransferase & WP_000376623.1 \\
\hline PaeAG1_03250 & EMC2982_03478 & 100.0 & Transposase TniB & $\operatorname{tniB} \Delta$ & $\begin{array}{l}\text { WP_003107582.1; } \\
\text { WP_021264342.1 }\end{array}$ \\
\hline PaeAG1_03251 & EMC2982_03477 & 100.0 & Transposase TniA & $\operatorname{tniA}$ & $\begin{array}{l}\text { WP_000179844.1; } \\
\text { YP_008766137.1 }\end{array}$ \\
\hline PaeAG1_03252 & EMC2982_03476 & 100.0 & Hypothetical protein & $\operatorname{urf} 2 \Delta$ & WP_000204520.1 \\
\hline PaeAG1_03253 & EMC2982_03475 & 100.0 & Mercury resistance protein merE & merE & $\begin{array}{c}\text { WP_000993386.1; } \\
\text { YP_789372.1 }\end{array}$ \\
\hline PaeAG1_03254 & EMC2982_03474 & 99.0 & Transcriptional regulator merD & merD & $\begin{array}{c}\text { WP_001277456.1; } \\
\text { YP_789373.1 }\end{array}$ \\
\hline PaeAG1_03255 & EMC2982_03473 & 99.8 & Mercuric reductase merA & merA & $\begin{array}{c}\text { WP_000105636.1; } \\
\text { YP_789374.1 }\end{array}$ \\
\hline PaeAG1_03256 & EMC2982_03472 & 100.0 & Transposase & $\operatorname{tnpA}$ & $\begin{array}{l}\text { WP_003111042.1; } \\
\text { WP_003460108.1 }\end{array}$ \\
\hline PaeAG1_03257 & EMC2982_03471 & 100.0 & TpnA repressor protein & $\operatorname{tnpC}$ & $\begin{array}{c}\text { WP_003111043.1; } \\
\text { NP_745109.1 }\end{array}$ \\
\hline PaeAG1_03258 & EMC2982_03470 & 100.0 & Hypothetical protein & $\mathrm{HP}$ & WP_003111045.1 \\
\hline PaeAG1_03259 & EMC2982_03469 & 100.0 & Hypothetical protein & $\mathrm{HP}$ & WP_003111046.1 \\
\hline PaeAG1_03260 & EMC2982_03468 & 100.0 & Homospermidine synthase (HPS) & HPS & WP_003111047.1 \\
\hline
\end{tabular}

Page 25/36 


\begin{tabular}{|c|c|c|c|c|c|}
\hline PaeAG1_03261 & EMC2982_03467 & 100.0 & Hypothetical protein & HP & WP_003111048.1 \\
\hline PaeAG1_03262 & EMC2982_03466 & 100.0 & Hypothetical protein & $\mathrm{HP}$ & WP_003111049.1 \\
\hline PaeAG1_03263** & EMC2982_03465 & 100.0 & Recombinase & Recombinase & WP_003111050.1 \\
\hline PaeAG1_03265** & EMC2982_03463 & 100.0 & Hypothetical protein & HP & WP_010792965.1 \\
\hline PaeAG1_03266 & EMC2982_03462 & 100.0 & Hypothetical protein & HP & WP_003092560.1 \\
\hline PaeAG1_03267 & EMC2982_03461 & 100.0 & Hypothetical protein PA2229 & PA2229 & $\begin{array}{c}\text { NP_250919.1; } \\
\text { WP_003113716.1 }\end{array}$ \\
\hline PaeAG1_03268 & EMC2982_03460 & 100.0 & Hypothetical protein PA2228 & PA2228 & $\begin{array}{c}\text { NP_250918.1; } \\
\text { WP_003113715.1 }\end{array}$ \\
\hline PaeAG1_03269 & EMC2982_03459 & 100.0 & AraC-type transcriptional regulator VqsM & vqsM (PA2227) & $\begin{array}{c}\text { NP_250917.1; } \\
\text { WP_003113714.1 }\end{array}$ \\
\hline
\end{tabular}

Notes:

* "EMC2982_" is the locus with our annotation (see Methods). See Supplementary Table S1 for locus in PGDB annotation file and amino-acid comparison against other genomes.

**PaeAG1_03264 is a tRNA, i.e. not included here.

Table 3. Annotation of protein-coding genes of the genomic region $\mathrm{GIC}_{\mathrm{IMP}-18}$ associated with the IMP-18-carrying integron 


\begin{tabular}{|c|c|c|c|c|c|}
\hline \multirow[t]{2}{*}{ PaeAG1 } & \multicolumn{2}{|l|}{ Pae-97 } & \multirow[t]{2}{*}{ Annotation } & \multirow[t]{2}{*}{ Name } & \multirow[t]{2}{*}{ RefSeq } \\
\hline & Gene* & Identity & & & \\
\hline PaeAG1_05736 & Pa97_05533 & 100.0 & Hypothetical protein PA4702 & PA4702 & $\begin{array}{c}\text { NP_253390.1; } \\
\text { WP_003095090.1 }\end{array}$ \\
\hline PaeAG1_05737 & Pa97_05534 & 100.0 & Hypothetical protein PA4703 & PA4703 & $\begin{array}{c}\text { NP_253391.1; } \\
\text { WP_003095094.1 }\end{array}$ \\
\hline PaeAG1_05738 & Pa97_05535 & 100.0 & cAMP-binding protein A PA4704, cbpA & $\begin{array}{c}\mathrm{cbpA} \\
(\mathrm{PA} 4704)\end{array}$ & $\begin{array}{c}\text { NP_253392.1; } \\
\text { WP_003095096.1 }\end{array}$ \\
\hline PaeAG1_05739 & Pa97_05536 & 100.0 & Recombinase & Recombinase & WP_023442562.1 \\
\hline PaeAG1_05740 & Pa97_05537 & 100.0 & $\begin{array}{c}\text { helix-turn-helix transcriptional regulator } \\
\text { (HTH-TR) }\end{array}$ & HTH-TR & WP_003148665.1 \\
\hline PaeAG1_05741 & Pa97_05538 & 99.8 & Hypothetical protein & $\mathrm{HP}$ & WP_137462639.1 \\
\hline PaeAG1_05742 & Pa97_05539 & 100.0 & Hypothetical protein & $\mathrm{HP}$ & WP_071567699.1 \\
\hline PaeAG1_05743 & Pa97_05540 & 100.0 & Hypothetical protein & $\mathrm{HP}$ & WP_042855636.1 \\
\hline PaeAG1_05744 & Pa97_05541 & 100.0 & $\begin{array}{c}\text { Type I restriction endonuclease subunit } \\
\mathrm{R}\end{array}$ & hsdR & $\begin{array}{l}\text { WP_042855635.1; } \\
\text { YP_005974822.1 }\end{array}$ \\
\hline PaeAG1_05745 & Pa97_05542 & 100.0 & Hypothetical protein & HP & WP_003148682.1 \\
\hline PaeAG1_05746 & Pa97_05543 & 100.0 & restriction endonuclease subunit S & hsdS & $\begin{array}{l}\text { WP_079393399.1; } \\
\text { YP_005974824.1 }\end{array}$ \\
\hline PaeAG1_05747 & Pa97_05544 & 100.0 & $\begin{array}{c}\text { type I restriction-modification system } \\
\text { (RMS) subunit M }\end{array}$ & hsdM & $\begin{array}{l}\text { WP_003148685.1; } \\
\text { YP_005974823.1 }\end{array}$ \\
\hline PaeAG1_05748 & Pa97_05545 & 100.0 & recombinase family protein & Recombinase & WP_003148687.1 \\
\hline PaeAG1_05749 & Pa97_05546 & 100.0 & class 1 integron integrase IntI1 & intI & YP_005221021.1 \\
\hline PaeAG1_05750 & $\begin{array}{l}\text { Pa97_05548 } \\
\text { (IMP-1) }\end{array}$ & 80.5 & $\begin{array}{c}\text { subclass B1 metallo-beta-lactamase } \\
\text { IMP-18 }\end{array}$ & IMP-18 & WP_060614779.1 \\
\hline PaeAG1_05750.1 & CP913_RS21750 & 36.4 & $\begin{array}{l}\text { DUF1010 domain-containing protein } \\
\text { gcuD }\end{array}$ & gcuD & WP_001336345.1 \\
\hline PaeAG1_05751 & Pa97_05547 & 36.4 & $\begin{array}{l}\text { oxacillin-hydrolyzing class D beta- } \\
\text { lactamase OXA-2 }\end{array}$ & OXA-2 & WP_034033256.1 \\
\hline PaeAG1_05751.1 & CP913_RS28765 & 99.4 & $\begin{array}{c}\text { Aminoglycoside } \mathrm{N}\left(6^{\prime}\right) \text {-acetyltransferase } \\
\text { type } 1 \text { aacA4 }\end{array}$ & aacA4 & WP_003159191.1 \\
\hline PaeAG1_05752 & Pa97_04840 & 100.0 & $\begin{array}{c}\text { sulfonamide-resistant dihydropteroate } \\
\text { synthase Sul1 }\end{array}$ & sul1 & WP_000259031.1 \\
\hline PaeAG1_05753 & Pa97_04839 & 100.0 & GNAT family N-acetyltransferase & GNAT & WP_000376623.1 \\
\hline PaeAG1_05754 & Pa97_05603 & 44.4 & ATP-binding protein, protease istD & istD & WP_000983249.1 \\
\hline PaeAG1_05755 & Pa97_04622 & 44.1 & Transposase istA & istA & $\begin{array}{l}\text { WP_001324342.1; } \\
\text { WP_000996451.1 }\end{array}$ \\
\hline PaeAG1_05756 & Pa97_05551 & 100.0 & Transposase TniB & $\operatorname{tniB} \Delta$ & $\begin{array}{l}\text { WP_003107582.1; } \\
\text { WP_021264342.1 }\end{array}$ \\
\hline PaeAG1_05757 & Pa97_05552 & 100.0 & Transposase TniA & $\operatorname{tniA}$ & $\begin{array}{l}\text { WP_000179844.1; } \\
\text { YP_008766137.1 }\end{array}$ \\
\hline PaeAG1_05758 & Pa97_05553 & 100.0 & Hypothetical protein & $\mathrm{HP}$ & WP_003157545.1 \\
\hline
\end{tabular}

Page 27/36 


\begin{tabular}{|c|c|c|c|c|c|}
\hline PaeAG1_05759 & Pa97_05554 & 99.6 & Hypothetical protein & $\mathrm{HP}$ & WP_003157546.1 \\
\hline PaeAG1_05760 & Pa97_05555 & 97.6 & iron(III) ABC transporter PhuW & phuW & $\begin{array}{c}\text { NP_253393.1; } \\
\text { WP_003113451.1 }\end{array}$ \\
\hline PaeAG1_05761 & Pa97_05556 & 99.6 & $\begin{array}{c}\text { heme ABC transporter ATP-binding } \\
\text { protein PhuV }\end{array}$ & phuV & $\begin{array}{c}\text { NP_253394.1; } \\
\text { WP_003095098.1 }\end{array}$ \\
\hline PaeAG1_05762 & Pa97_05557 & 100.0 & iron $\mathrm{ABC}$ transporter permease $\mathrm{PhuU}$ & phuU & $\begin{array}{c}\text { NP_253395.1; } \\
\text { WP_003121063.1 }\end{array}$ \\
\hline
\end{tabular}

Notes:

* "Pa97_" is the locus with our annotation (see Methods). See Supplementary Table S2 for locus in PGDB annotation file and amino-acid comparison against other genomes. Cases with "CP913_" locus refers to the PGDB annotation file with a better score due to annotation algorithms differences.

Table 4. Comparison of different IMP-18-carrying integrons

\begin{tabular}{|c|c|c|c|c|c|c|}
\hline \multicolumn{7}{|c|}{ List of numbered class 1 IMP-18 carrying integrons } \\
\hline $\begin{array}{c}\text { In } \\
\text { names }\end{array}$ & $\begin{array}{c}\text { GenBank Access } \\
\text { Number }\end{array}$ & $\begin{array}{c}\text { Gene cassette } \\
1\end{array}$ & $\begin{array}{c}\text { Gene cassette } \\
2\end{array}$ & $\begin{array}{c}\text { Gene cassette } \\
3\end{array}$ & $\begin{array}{c}\text { Gene cassette } \\
4\end{array}$ & $\begin{array}{c}\text { Gene cassette } \\
5\end{array}$ \\
\hline In96 & EF184215 & IMP-18 & aadA1b & - & - & - \\
\hline In98 & AB587676 & aаcA7 & qacF4 & IMP-18 & gсu39 & aadA1b \\
\hline In 169 & HM138673 & IMP-18 & aadA1b & OXA-2 & aadA1b & - \\
\hline In 614 & AB587676 & aаcA7 & qacF4 & IMP-18 & gсu39 & $a a d A 1 b$ \\
\hline In706 & JN624388 & IMP-18 & aаc $A 43 b$ & aаcA45 & OXA-2 & $\operatorname{gcuD}$ \\
\hline In707 & JN596991 & IMP-18 & $a a d A 1 b$ & OXA-224 & - & - \\
\hline $\begin{array}{c}\text { New } \\
\text { In1666 }\end{array}$ & KC907377 & IMP-18 & $g c u D$ & OXA-2 & aacA4 & - \\
\hline
\end{tabular}

\section{Figures}




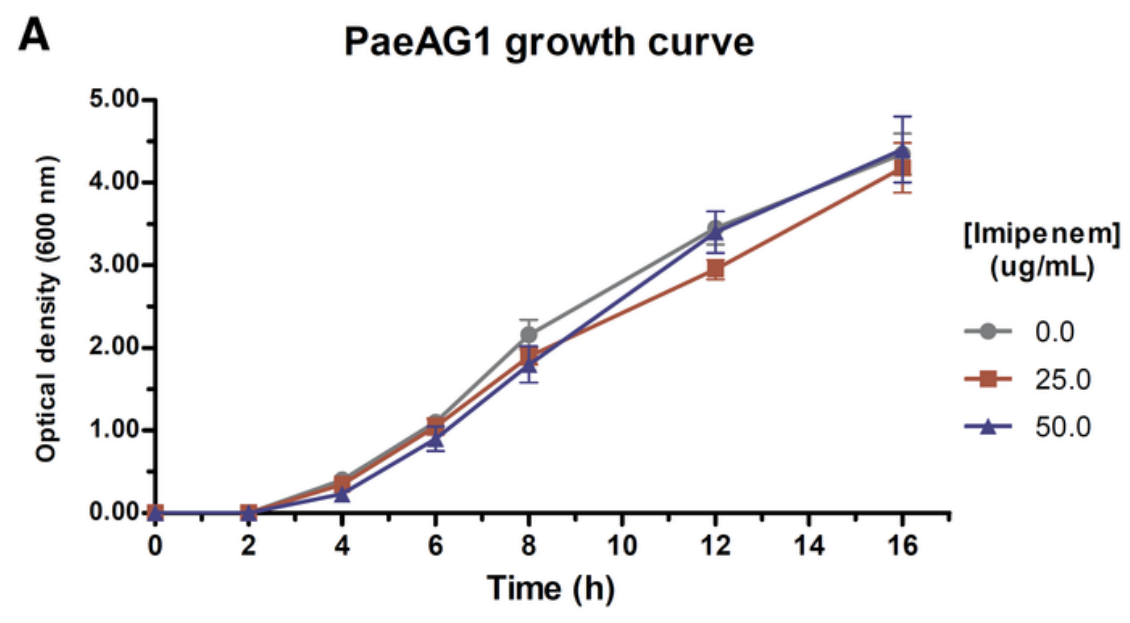

B

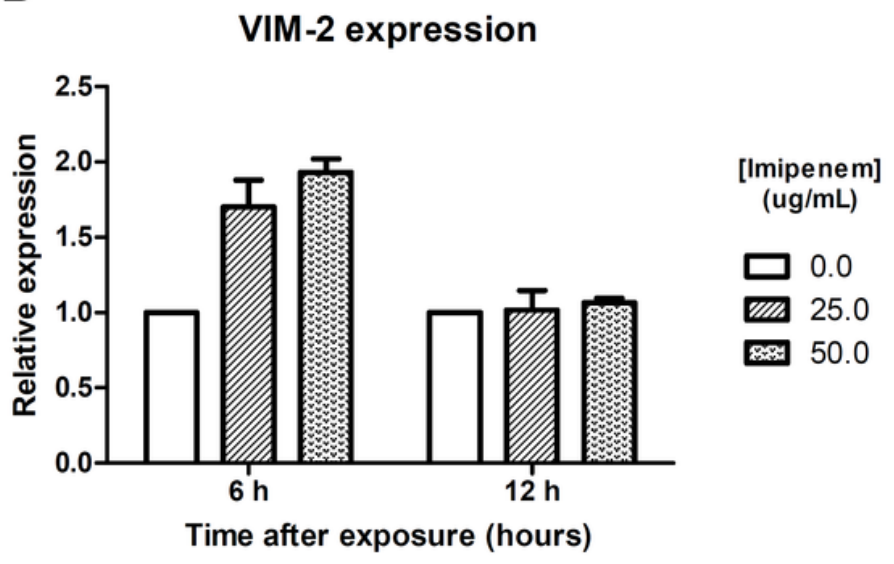

C

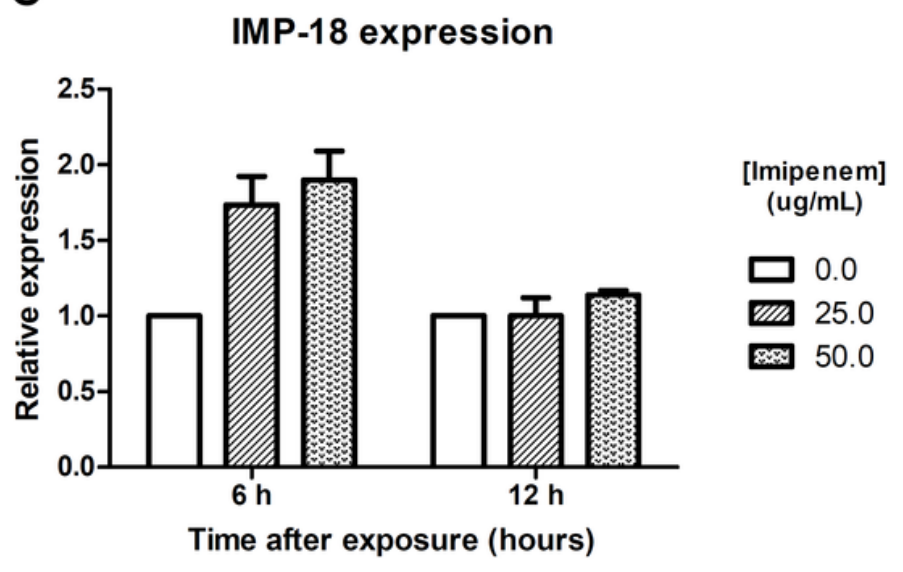

\section{Figure 1}

VIM-2 and IMP-18 expression after imipenem exposure. A RT-qPCR was performed to assess the transcriptomic activity of VIM-2 and IMP-18 genes. PaeAG1 was exposed to two imipenem concentrations, showing no effects on the growth curves (A). Relative gene expression showed that higher induction occurs at 6 hours after exposure, not only for VIM-1 (B) but also for IMP-18 (C). Relative expression was statistically different by time but not by concentration $(p<0.05)$. 


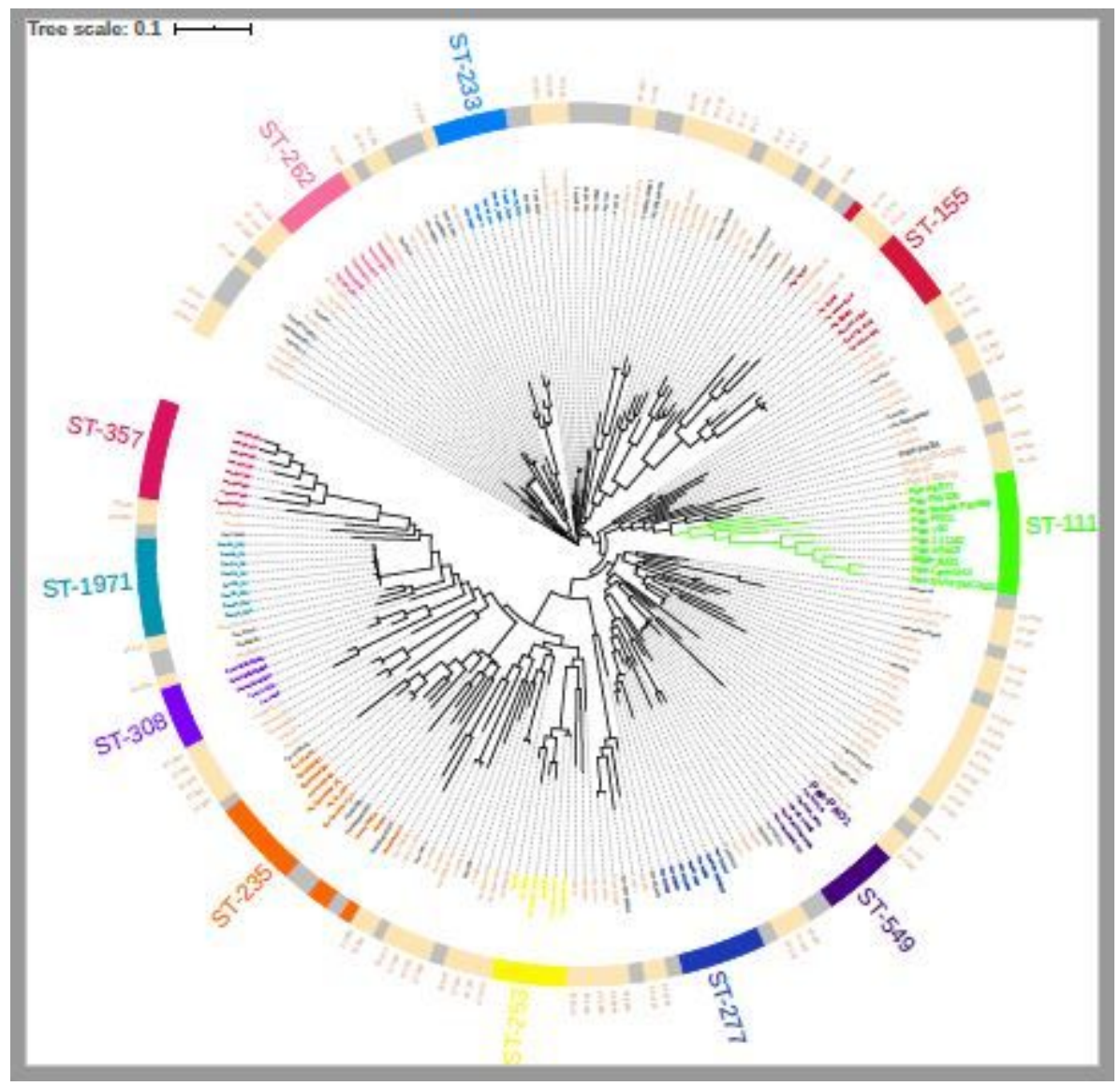

Figure 2

Comparative genomic analysis of $211 \mathrm{P}$. aeruginosa strains. By a pan-genome analysis strategy, the complete genomes were compared and the gene composition defined groups that can be described in turn by the MLST genotyping profile. ST groups with a low frequency of fewer than 5 strains are shown in beige and cases with unknown ST were represented in gray. ST groups with 5 or more strains were represented with colors. The Pae-AG1 strain and all the other ST-111 strains are located in a separated cluster, as shown in green. 


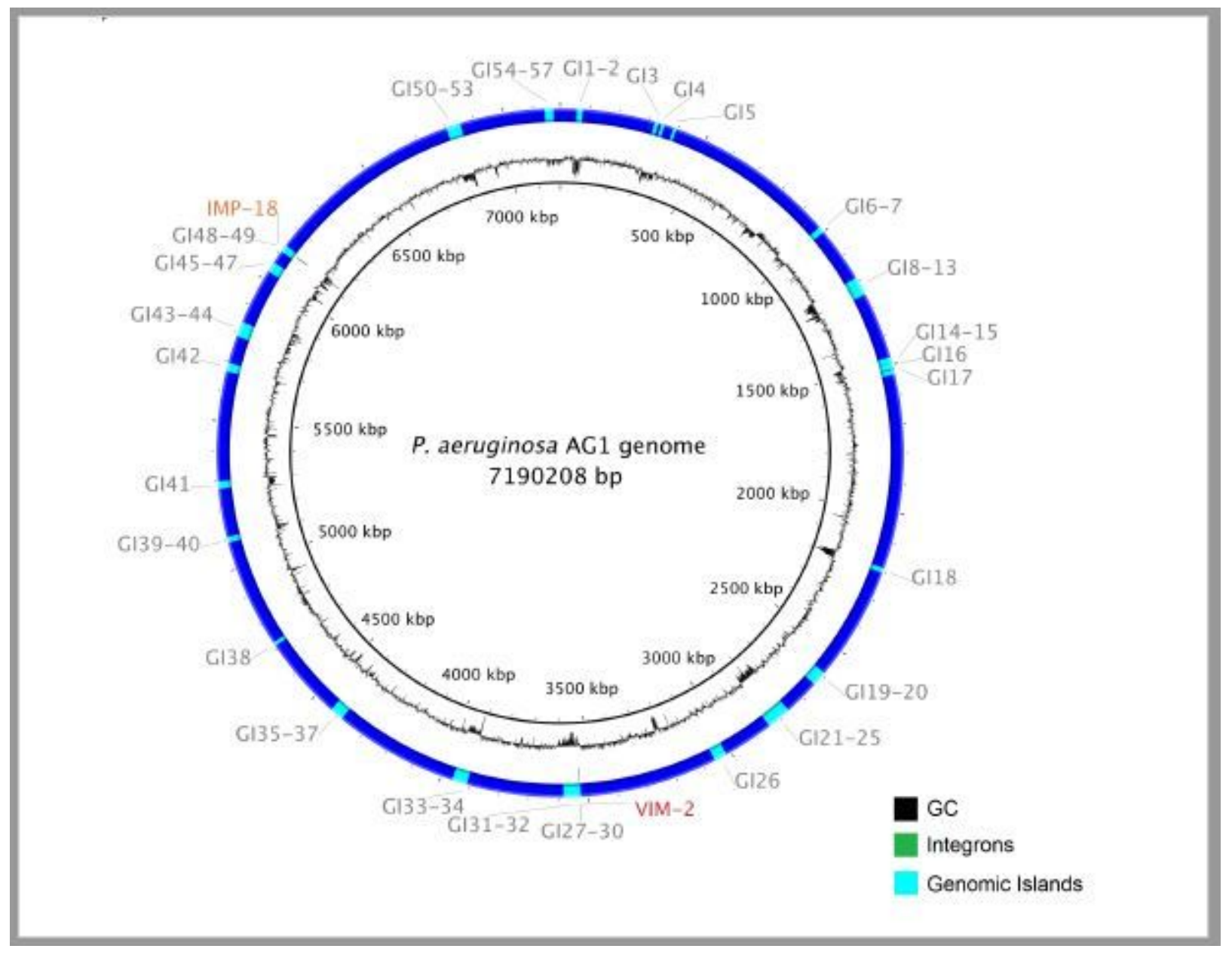

\section{Figure 3}

Distribution of genomic islands of PaeAG1 along the genome. The 57 predicted genomic islands are distributed along the PaeAG1 genome, and most of them forming groups with two or more islands in a row (genomic islands clusters, GIC), which are jointly named in a single label. 


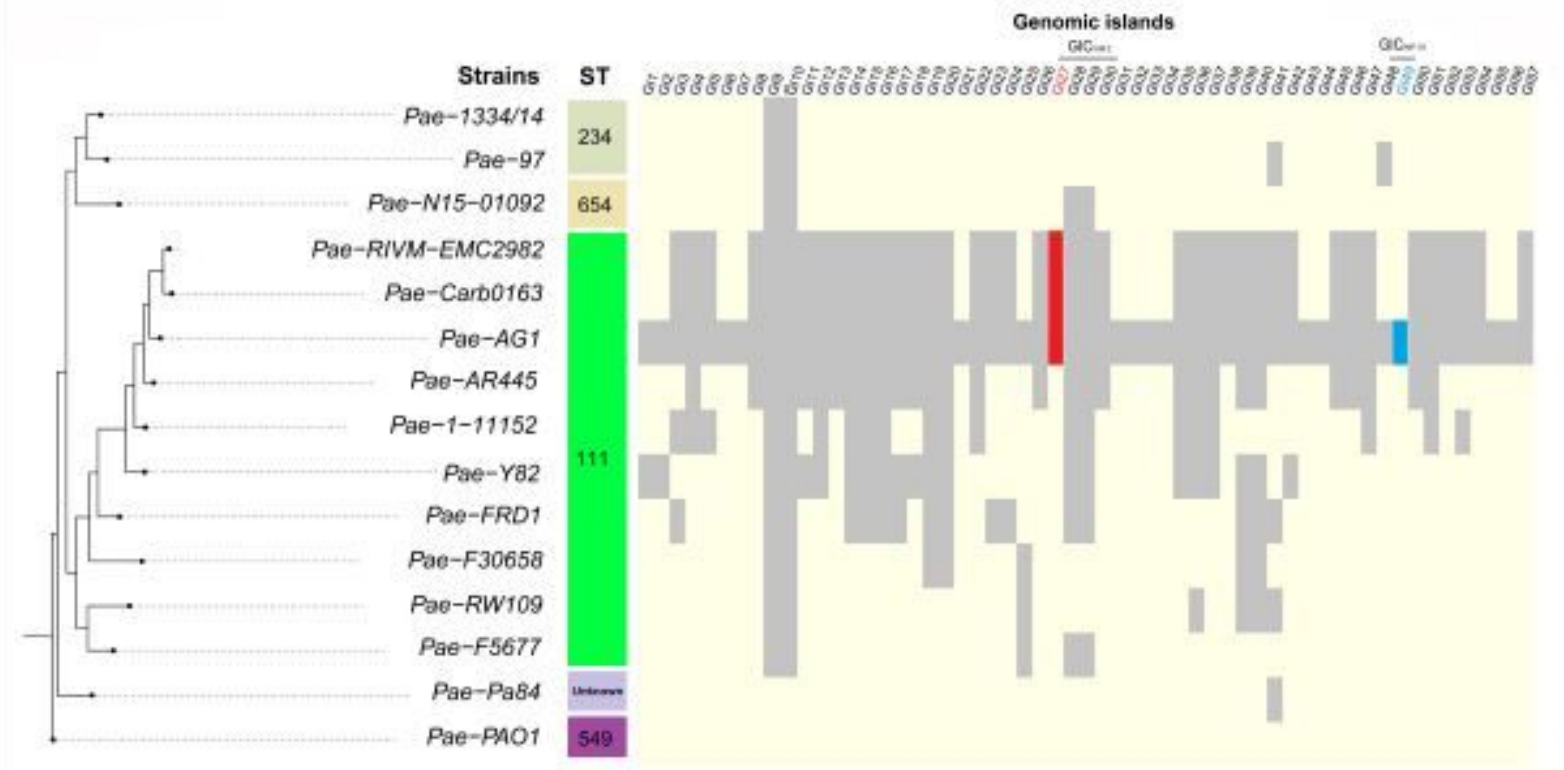

\section{Figure 4}

Comparative analysis of the presence/absence of PaeAG1 genomic islands in other ST-111 strains and representative genomes. The 57 genomic islands were searched in the genomes of the other ST-111 strains, the reference strain PAO1 (ST-549), and three other strains close to the ST-111 group (see Fig. 2). The GI27 genomic island includes the VIM-2-carrying integron and it is present in PaeAG1 and two other ST-111 strains, while the GI49 (blue) harboring IMP-18-carrying integron is unique to PaeAG1 and is not it is present in none of the other 210 strains in the study. Other genomic islands linked to GICVIM-2 and GICIMP-18 have a different pattern of occurrence between strains. 


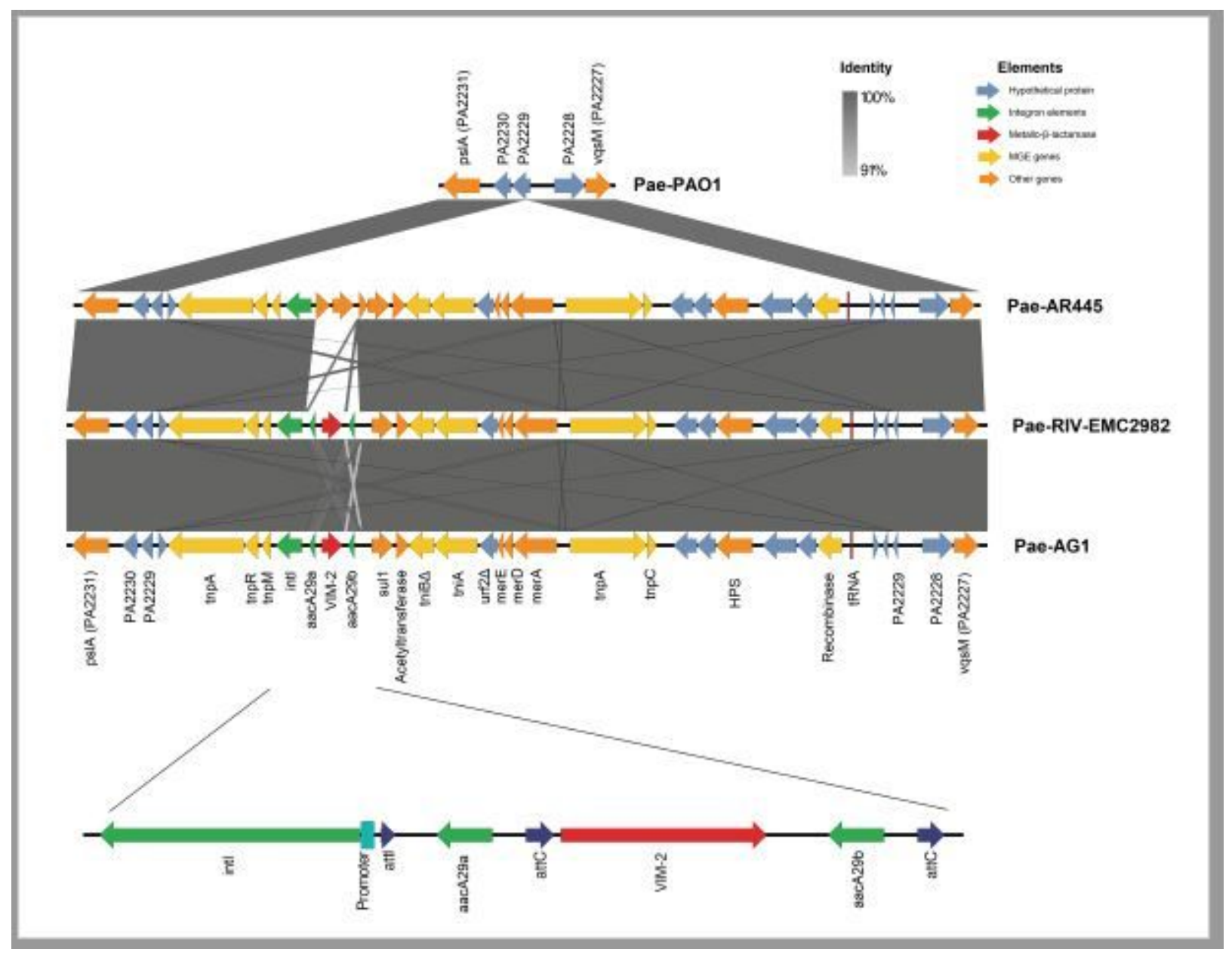

\section{Figure 5}

Description of the architecture of the genomic region GICVIM-2 containing the old-acquaintance VIM-2carrying integron. The genomic region GICVIM-2 is absent in the reference sequence Pae-PAO1, meanwhile it is mostly present in Pae-AR445, but without most of the integron. Full coverage of the region was identified in Pae-RIV-EMC2982. The architecture of the VIM-2-carrying integron is shown. 


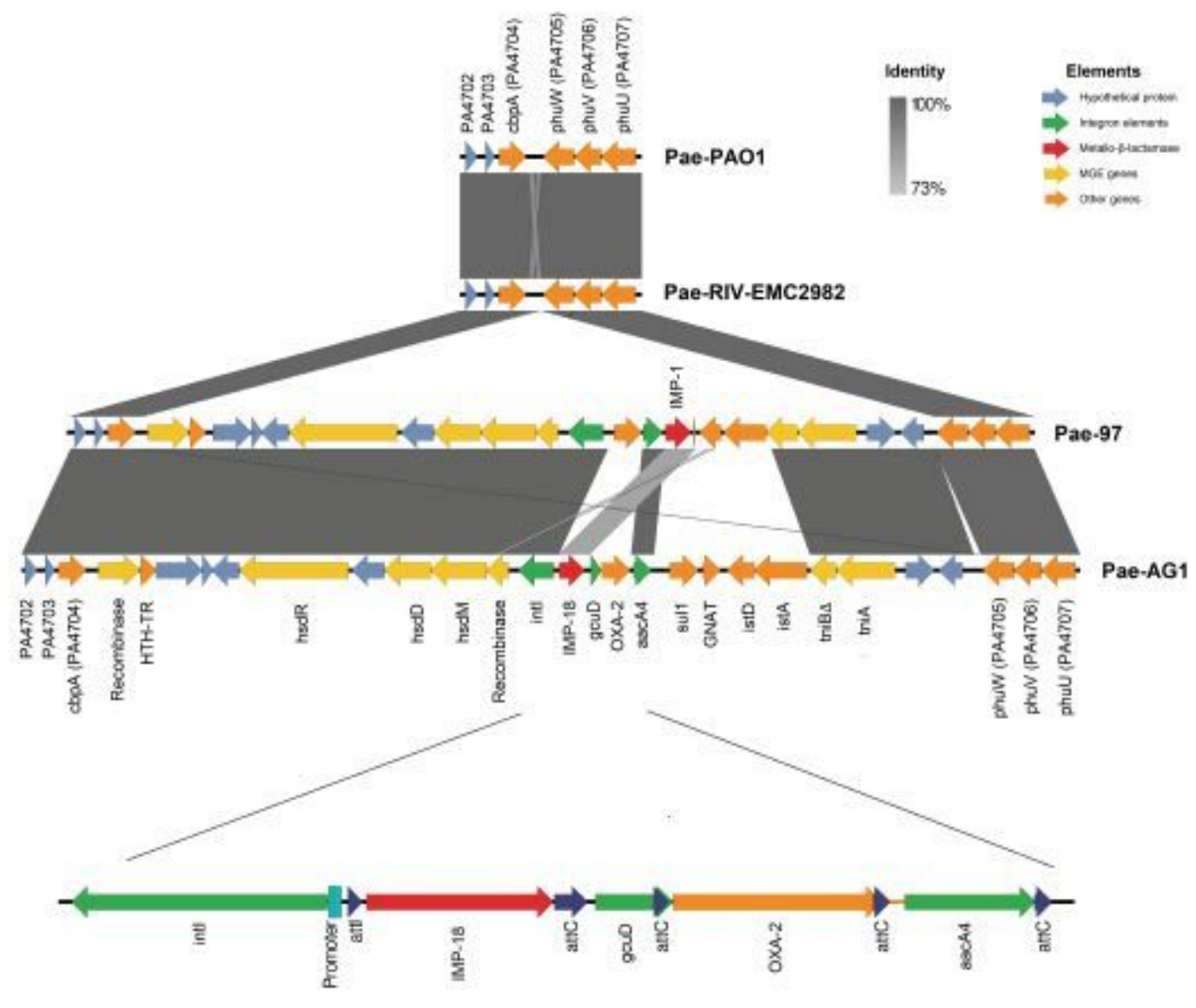

Figure 6

Description of the architecture of the exclusive genomic region GICIMP-18 containing the new IMP-18carrying integron. The genomic region GICIMP-18 is absent in the reference sequence Pae-PAO1 and PaeRIV-EMC2982 strains, meanwhile it is partially present in Pae-97. The architecture of the IMP-18-carrying integron is shown with an arrangement that is reported here for the first time. 
A

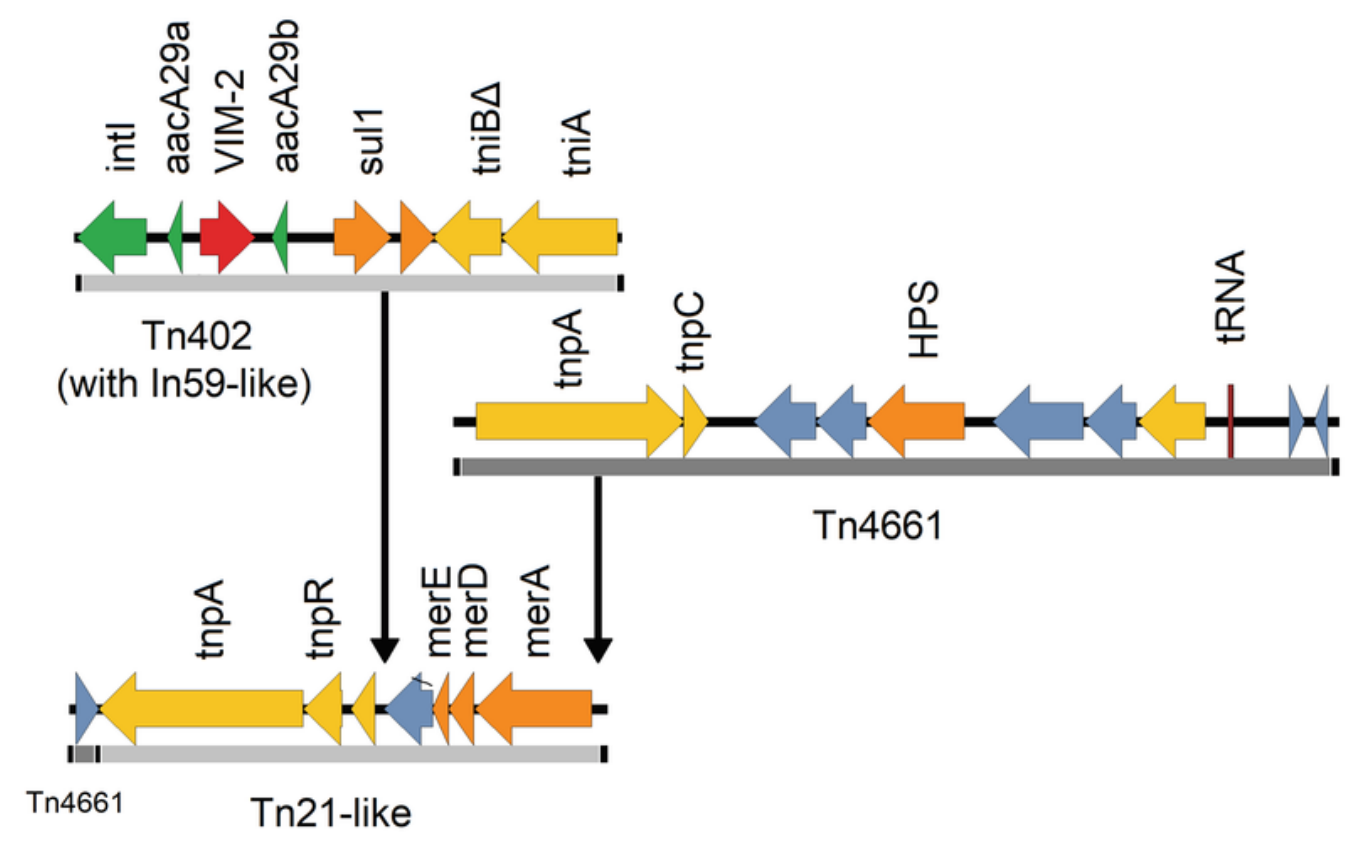

B
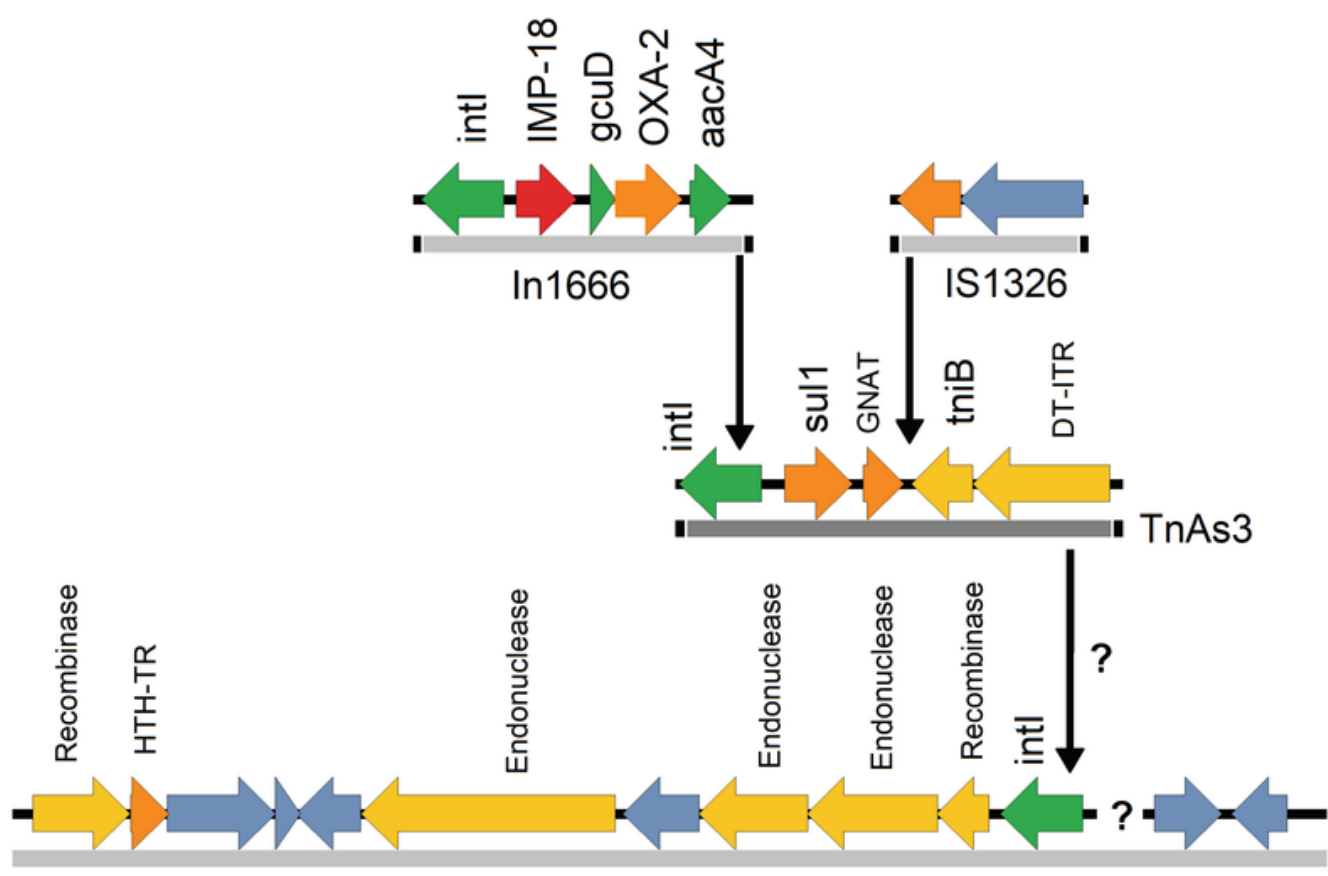

Tn?

Figure 7

Possible evolutionary steps associated with the genomic regions of the VIM-2- and IMP-18-carrying integrons. Different mobile elements are involved in the current state of the genomic region, being completely described for GICVIM-2 (A) and partially for GICIMP-18 (B).

\section{Supplementary Files}


This is a list of supplementary files associated with this preprint. Click to download.

- SupplementaryTableS1.docx

- SupplementaryTableS2.xlsx

- SupplementaryTableS3.xlsx

- SupplementaryTableS4.xlsx

- Supplementaryfile1Allstrainsinformation.xlsx

- Supplementaryfile2Pangenomeanalysisresults.xlsx 